\title{
Impact of the Interfacial Molecular Structure Organization on the Charge Transfer State Formation and Exciton Delocalization in Merocyanine: $\mathrm{PC}_{61} \mathrm{BM}$ Blends
}

\author{
K. Budzinauskas ${ }^{*}$, D. Fazzi², D. Hertel2 ${ }^{2}$, S. Rüth², J. Schelter², P. Weitkamp², S. Diesing² K. \\ Meerholz*2 , Paul H.M. Van Loosdrecht*1 \\ ${ }^{1}$ Institute of Physics 2, University of Cologne, Zülpicher Str.77, Cologne, Germany \\ 2Department Chemistry, Institute of Physical Chemistry, University of Cologne, Greinstrasse 4-6, Cologne, \\ Germany
}

\section{Synthesis of ML merocyanine}

2,3,3-trimethylindolenine $(5 \mathrm{~g}, 31.4 \mathrm{mmol})$ and 1-iodononane $(7.98 \mathrm{~g}, 31.4 \mathrm{mmol})$ were heated under an argon atmosphere to $120{ }^{\circ} \mathrm{C}$ for 24 hours. After cooling to room temperature the residue was dissolved in dichloromethane and a $1 \mathrm{M}$ aqueous solution of potassium hydroxide $(90 \mathrm{ml})$ was added. The mixture was stirred at room temperature for $1 \mathrm{~h}$ and the crude product (1-heptyl-3,3-dimethyl-2-methyleneindoline) was extracted with dichloromethane. After drying the organic phase over $\mathrm{MgSO}_{4}$ the solvent was removed under reduced pressure. The crude product was used immediately without any further purification.

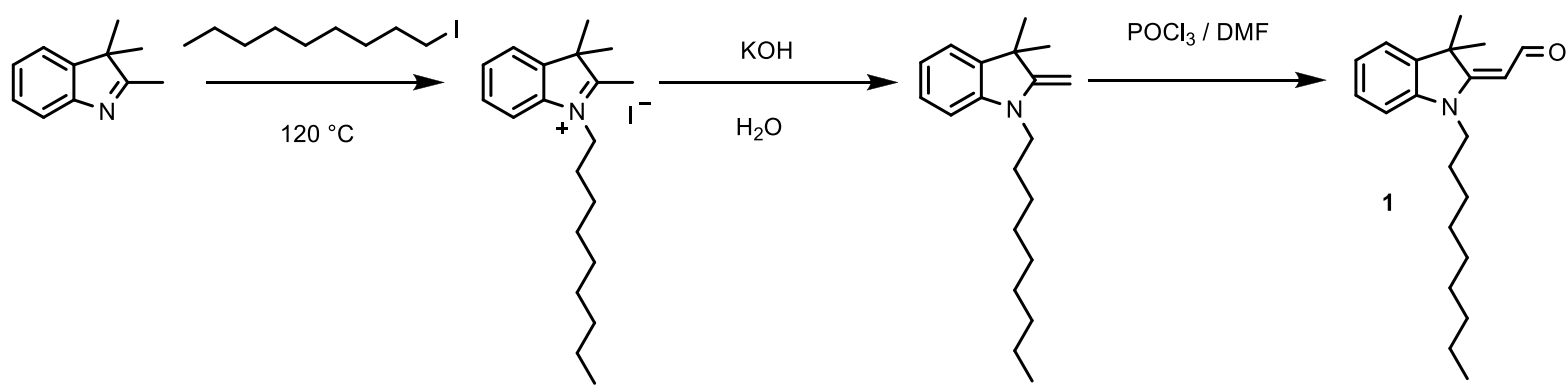

Figure S1 Synthesis of (E)-2-(3,3-dimethyl-1-nonylindolin-2-ylidene)acetaldehyde (1).

Under an argon atmosphere dry dimethylformamide $(5.5 \mathrm{ml})$ was cooled to $0{ }^{\circ} \mathrm{C}$ and phosphorous oxychloride (5.37 g, $35.0 \mathrm{mmol})$ was slowly added. The crude 3,3-dimethyl-1-nonyl-2-methyleneindoline was dissolved in dry dimethylformamide $(7.5 \mathrm{ml})$ and added dropwise to the $\mathrm{POCl}_{3} / \mathrm{DMF}$ mixture. The reaction mixture was stirred at $40{ }^{\circ} \mathrm{C}$ for $1 \mathrm{~h}$ and then added to water containing ice $(30 \mathrm{ml})$. After adjusting the $\mathrm{pH}$ to 10 with a diluted sodium hydroxide solution the mixture was heated under reflux for 30 minutes. The crude product was extracted with dichloromethane, the organic phase was dried over $\mathrm{MgSO}_{4}$ and the solvent was removed under reduced pressure.

Column chromatography on silica with toluene / ethyl acetate (5:1) as eluent yielded a red oil (7.36 g, 23.5 mmol, $75 \%$ ).

${ }^{1} \mathrm{H}$ NMR (acetone-d $\left.6,300 \mathrm{MHz}\right): \delta / \mathrm{ppm}=10.00\left(1 \mathrm{H}, \mathrm{d},{ }^{3} \mathrm{~J}=8.5 \mathrm{~Hz}\right) ; 7.38(1 \mathrm{H}, \mathrm{m}) ; 7.27(1 \mathrm{H}, \mathrm{m}) ; 7.07-6.99$ $(2 \mathrm{H}) ; 5.33\left(1 \mathrm{H}, \mathrm{d},{ }^{3} J=8.5 \mathrm{~Hz}\right) ; 3.78\left(2 \mathrm{H}, \mathrm{t},{ }^{3} \mathrm{~J}=7.5 \mathrm{~Hz}\right) ; 1.70(2 \mathrm{H}, \mathrm{m}) ; 1.65(6 \mathrm{H}, \mathrm{s}) ; 1.50-1.23(12 \mathrm{H}) ; 0.86$ $(3 \mathrm{H}, \mathrm{m})$. 

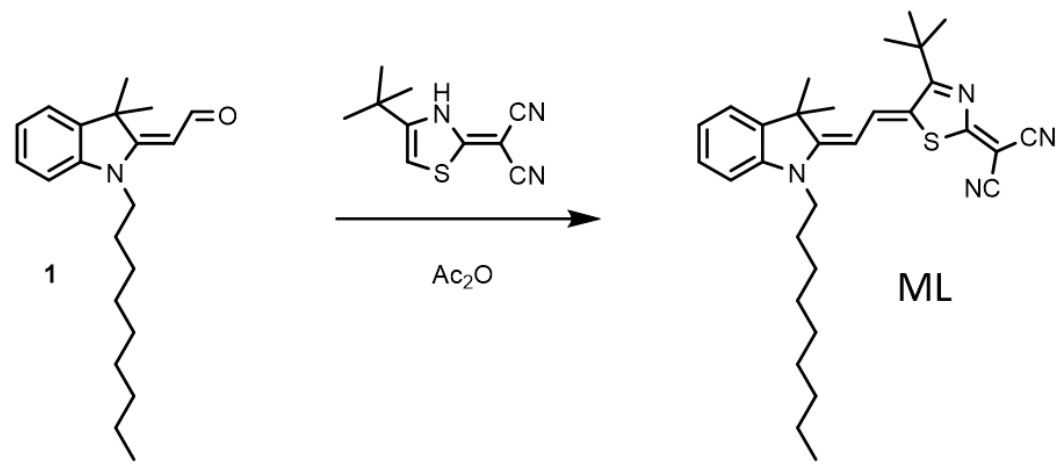

Figure S2 Synthesis of 2-((Z)-4-tert-butyl-5-((E)-2-(3,3-dimethyl-1-nonylindolin-2-ylidene)ethylidene)thiazol2(5H)-ylidene)malononitrile (ML).

A mixture of 1 (1.76 g, $5.62 \mathrm{mmol})$, 2-(4-tert-butylthiazol-2(3H)-ylidene)malononitrile ${ }^{[1]}(1.05 \mathrm{~g}, 5.09 \mathrm{mmol})$ and acetic anhydride $(6 \mathrm{ml})$ was heated to $90{ }^{\circ} \mathrm{C}$ for $1 \mathrm{~h}$. After cooling to room temperature $\mathrm{n}$-hexane was added and the precipitate was collected by filtration, washed with 2-propanol and n-hexane and dried in vacuo. Subsequent purification by column chromatography on silica with dichloromethane / ethyl acetate (99: 1) as eluent yielded a blue powder (1.47 g, $2.94 \mathrm{mmol}, 52 \%)$.

${ }^{1} \mathrm{H}$ NMR $\left(\mathrm{CD}_{2} \mathrm{Cl}_{2}, 500 \mathrm{MHz}\right): \delta / \mathrm{ppm}=8.33\left(1 \mathrm{H}, \mathrm{d},{ }^{3} J=13.4 \mathrm{~Hz}\right) ; 7.45-7.34(2 \mathrm{H}), 7.25(1 \mathrm{H}, \mathrm{m}), 7.09(1 \mathrm{H}$, $\mathrm{m}), 5.65\left(1 \mathrm{H}, \mathrm{d},{ }^{3} \mathrm{~J}=13.4 \mathrm{~Hz}\right), 3.94\left(2 \mathrm{H}, \mathrm{t},{ }^{3} \mathrm{~J}=7.3 \mathrm{~Hz}\right), 1.81(2 \mathrm{H}, \mathrm{m}), 1.69(6 \mathrm{H}, \mathrm{s}), 1.55(9 \mathrm{H}, \mathrm{s}), 1.48-1.21$ $(12 \mathrm{H}), 0.87(3 \mathrm{H}, \mathrm{m}) .{ }^{13} \mathrm{C} \mathrm{NMR}\left(\mathrm{CD}_{2} \mathrm{Cl}_{2}, 125 \mathrm{MLz}\right): \delta / \mathrm{ppm}=185.0\left(\mathrm{C}_{\mathrm{q}}\right), 179.4\left(\mathrm{C}_{\mathrm{q}}\right), 172.4\left(\mathrm{C}_{\mathrm{q}}\right), 142.4\left(\mathrm{C}_{\mathrm{q}}\right)$, $140.6\left(\mathrm{C}_{\mathrm{q}}\right), 138.3(\mathrm{CH}), 128.9(\mathrm{CH}), 126.5\left(\mathrm{C}_{\mathrm{q}}\right), 125.2(\mathrm{CH}), 122.5(\mathrm{CH}), 118.2\left(\mathrm{C}_{\mathrm{q}}\right), 116.1\left(\mathrm{C}_{\mathrm{q}}\right), 110.7(\mathrm{CH})$, $99.2(\mathrm{CH}), 55.9\left(\mathrm{C}_{\mathrm{q}}\right), 49.1\left(\mathrm{C}_{\mathrm{q}}\right), 44.5\left(\mathrm{CH}_{2}\right), 38.1\left(\mathrm{C}_{\mathrm{q}}\right), 32.1\left(\mathrm{CH}_{2}\right), 31.4\left(\mathrm{CH}_{3}\right), 29.5\left(3 \mathrm{xCH}_{2}\right), 28.4\left(\mathrm{CH}_{3}\right), 27.3$ $\left(\mathrm{CH}_{2}\right), 27.2\left(\mathrm{CH}_{2}\right), 22.9\left(\mathrm{CH}_{2}\right), 14.1\left(\mathrm{CH}_{3}\right)$. HR-ESI-MS $(\mathrm{MeCN})$ : calc. $\mathrm{m} / \mathrm{z}=500.29682$ for $\mathrm{C}_{31} \mathrm{H}_{40} \mathrm{~N}_{4} \mathrm{~S}$, found $\mathrm{m} / \mathrm{z}=500.29739, \Delta=1.14 \mathrm{ppm}$.

\section{Synthesis of MB merocyanine}

2,3,3-trimethylindolenine ( $2.26 \mathrm{~g}, 14.2 \mathrm{mmol})$ and 1-iodo-2-ethylhexane ( $3.41 \mathrm{~g}, 14.2 \mathrm{mmol})$ were heated under an argon atmosphere to $120{ }^{\circ} \mathrm{C}$ for 24 hours. After cooling to room temperature the residue was dissolved in dichloromethane and a $1 \mathrm{M}$ aqueous solution of potassium hydroxide $(90 \mathrm{ml})$ was added. The mixture was stirred at room temperature for $1 \mathrm{~h}$ and the crude product (1-(2-ethylhexyl)-3,3-dimethyl-2-methyleneindoline) was extracted with dichloromethane. After drying the organic phase over $\mathrm{MgSO}_{4}$ the solvent was removed under reduced pressure. The crude product was used immediately without any further purification.

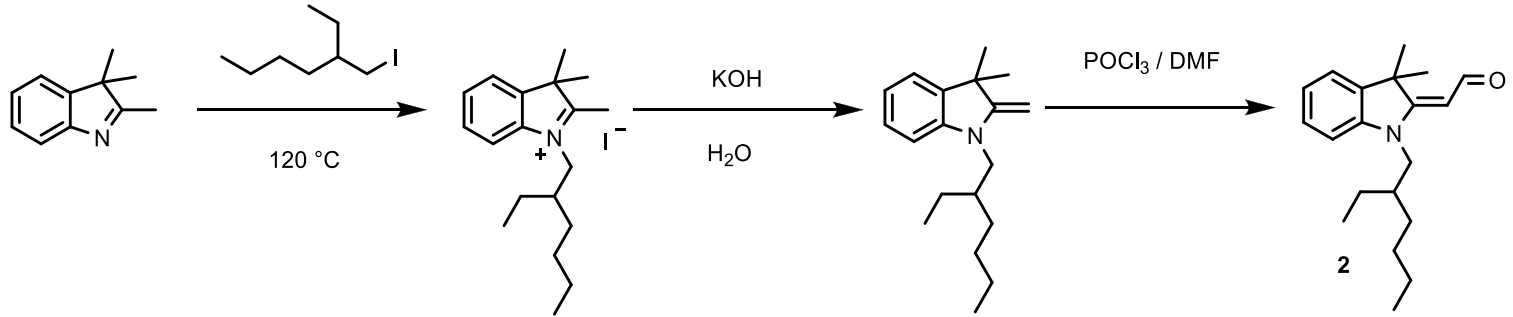

Figure S3 Synthesis of (E)-2-(3,3-dimethyl-1-(2-ethylhexyl)indolin-2-ylidene)acetaldehyde (2).

Under an argon atmosphere dry dimethylformamide $(3.5 \mathrm{ml})$ was cooled to $0{ }^{\circ} \mathrm{C}$ and phosphorous oxychloride (2.43 g, $15.8 \mathrm{mmol}$ ) was slowly added. The crude 3,3-dimethyl-1-(2-ethylhexyl)-2-methyleneindoline was dissolved in dry dimethylformamide $(4.5 \mathrm{ml})$ and added dropwise to the $\mathrm{POCl}_{3} / \mathrm{DMF}$ mixture. The reaction mixture was stirred at $40{ }^{\circ} \mathrm{C}$ for $1 \mathrm{~h}$ and then added to water containing ice $(30 \mathrm{ml})$. After adjusting the $\mathrm{pH}$ to 10 
with a diluted sodium hydroxide solution the mixture was heated under reflux for 30 minutes. The crude product was extracted with dichloromethane, the organic phase was dried over $\mathrm{MgSO}_{4}$ and the solvent was removed under reduced pressure.

Column chromatography on silica with toluene / ethyl acetate (7:1) as eluent yielded a red oil (0.92 g, 3.07 mmol, $22 \%)$.

${ }^{1} \mathrm{H} \mathrm{NMR}\left(\mathrm{CDCl}_{3}, 300 \mathrm{MHz}\right): \delta / \mathrm{ppm}=10.01\left(1 \mathrm{H}, \mathrm{d},{ }^{3} J=9.0 \mathrm{~Hz}\right) ; 7.29-7.21(2 \mathrm{H}) ; 7.05(1 \mathrm{H}, \mathrm{m}) ; 6.84(1 \mathrm{H}$, m); $5.42\left(1 \mathrm{H}, \mathrm{d},{ }^{3} J=9.0 \mathrm{~Hz}\right) ; 3.57\left(2 \mathrm{H}, \mathrm{d},{ }^{3} \mathrm{~J}=7.7 \mathrm{~Hz}\right) ; 1.96(1 \mathrm{H}, \mathrm{m}) ; 1.66(6 \mathrm{H}, \mathrm{s}) ; 1.41-1.21(8 \mathrm{H}) ; 0.91(3 \mathrm{H}$, $\mathrm{m}) ; 0.85(3 \mathrm{H}, \mathrm{m})$.

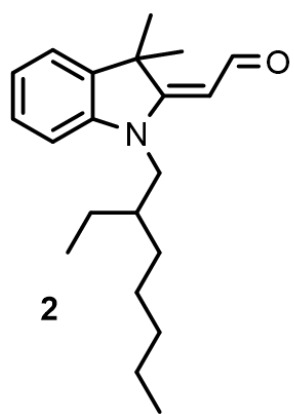

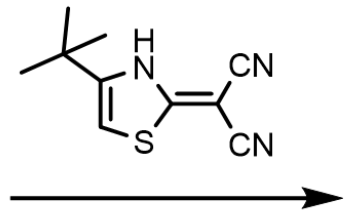

$\mathrm{Ac}_{2} \mathrm{O}$

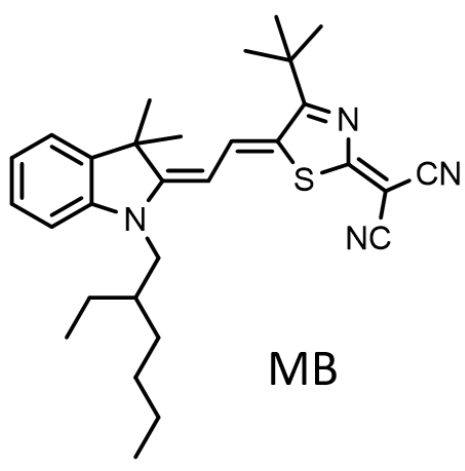

Figure S4 Synthesis of 2-((Z)-4-tert-butyl-5-((E)-2-(3,3-dimethyl-1-(2-ethylhexyl)indolin-2ylidene)ethylidene)thiazol-2(5H)-ylidene)malononitrile (MB).

A mixture of 2 (0.92 g, $2.25 \mathrm{mmol})$, 2-(4-tert-butylthiazol-2(3H)-ylidene)malononitrile ${ }^{[1]}(0.42 \mathrm{~g}, 2.04 \mathrm{mmol})$ and acetic anhydride $(2.3 \mathrm{ml})$ was heated to $90{ }^{\circ} \mathrm{C}$ for $1 \mathrm{~h}$. After cooling to room temperature n-hexane was added and the precipitate was collected by filtration, washed with 2-propanol and n-hexane and dried in vacuo. Subsequent purification by column chromatography on silica with dichloromethane as eluent yielded a blue powder $(0.59 \mathrm{~g}, 1.21 \mathrm{mmol}, 54 \%)$.

${ }^{1} \mathrm{H}$ NMR $\left(\mathrm{CD}_{2} \mathrm{Cl}_{2}, 500 \mathrm{MHz}\right): \delta / \mathrm{ppm}=8.31\left(1 \mathrm{H}, \mathrm{d},{ }^{3} J=13.4 \mathrm{~Hz}\right) ; 7.42-7.34(2 \mathrm{H}), 7.24(1 \mathrm{H}, \mathrm{m}), 7.08(1 \mathrm{H}$, $\mathrm{m}), 5.66\left(1 \mathrm{H}, \mathrm{d},{ }^{3} J=13.4 \mathrm{~Hz}\right), 3.84(2 \mathrm{H}, \mathrm{m}), 1.98(1 \mathrm{H}, \mathrm{m}), 1.69(6 \mathrm{H}, \mathrm{s}), 1.55(9 \mathrm{H}, \mathrm{s}), 1.49-1.25(8 \mathrm{H}), 0.98$ $\left(3 \mathrm{H}, \mathrm{t},{ }^{3} \mathrm{~J}=7.4 \mathrm{~Hz}\right) ; 0.90\left(3 \mathrm{H}, \mathrm{t},{ }^{3} \mathrm{~J}=7.2 \mathrm{~Hz}\right) .{ }^{13} \mathrm{C}$ NMR APT $\left(\mathrm{CD}_{2} \mathrm{Cl}_{2}, 125 \mathrm{MLz}\right): \delta / \mathrm{ppm}=185.0\left(\mathrm{C}_{\mathrm{q}}\right), 179.4$ $\left(\mathrm{C}_{\mathrm{q}}\right), 172.9\left(\mathrm{C}_{\mathrm{q}}\right), 143.1\left(\mathrm{C}_{\mathrm{q}}\right), 140.6\left(\mathrm{C}_{\mathrm{q}}\right), 138.2(\mathrm{CH}), 128.9(\mathrm{CH}), 126.7\left(\mathrm{C}_{\mathrm{q}}\right), 125.1(\mathrm{CH}), 122.5(\mathrm{CH}), 118.0$ $\left(\mathrm{C}_{\mathrm{q}}\right), 116.0\left(\mathrm{C}_{\mathrm{q}}\right), 111.0(\mathrm{CH}), 99.9(\mathrm{CH}), 55.9\left(\mathrm{C}_{\mathrm{q}}\right), 49.1\left(\mathrm{C}_{\mathrm{q}}\right), 48.6\left(\mathrm{CH}_{2}\right), 38.4(\mathrm{CH}), 31.5\left(\mathrm{CH}_{3}\right), 31.2\left(\mathrm{CH}_{2}\right)$ $31.1\left(\mathrm{C}_{\mathrm{q}}\right), 29.0\left(\mathrm{CH}_{2}\right), 28.3\left(\mathrm{CH}_{3}\right), 24.7\left(\mathrm{CH}_{2}\right), 23.2\left(\mathrm{CH}_{2}\right), 14.1\left(\mathrm{CH}_{3}\right), 11.1\left(\mathrm{CH}_{3}\right)$. HR-ESI-MS $(\mathrm{MeCN})$ : calc. $\mathrm{m} / \mathrm{z}=486.28117$ for $\mathrm{C}_{30} \mathrm{H}_{38} \mathrm{~N}_{4} \mathrm{~S}$, found $\mathrm{m} / \mathrm{z}=486.28029, \Delta=1.81 \mathrm{ppm}$.

\section{References}

[1] H. Bürckstümmer, E. V Tulyakova, M. Deppisch, M. R. Lenze, N. M. Kronenberg, M. Gsänger, M. Stolte, K. Meerholz, F. Würthner, Angew. Chem. Int. Ed. 2011, 50, 11628-11632.

\section{Device characteristics}

All solar cells were fabricated on indium-tin oxide (ITO, $125 \mathrm{~nm}$ ) coated glass. The substrates were exposed to ozone for $3 \mathrm{~min}$ and transferred to the evaporation chamber to evaporate $10 \mathrm{~nm} \mathrm{MoO}$. After transfer to a glove box active layers of about $70 \mathrm{~nm}$ were spin-coated from chloroform solutions of 13 to $15 \mathrm{mg} / \mathrm{mL}$ mixed in a 
ratio of 2:3 ( $\mathrm{MC}$ to $\mathrm{PC}_{61} \mathrm{BM}$ ). Neat film was prepared without $\mathrm{PC}_{61} \mathrm{BM}$ in the same way. The device fabrication was completed by thermal evaporation of $5 \mathrm{~nm} \mathrm{Ca}$ and $120 \mathrm{~nm} \mathrm{Ag}$ for solar cells or $10 \mathrm{~nm} \mathrm{MoO}_{3}$ and $120 \mathrm{~nm}$ $\mathrm{Ag}$ for unipolar diodes (SCLC).

The solar cell characteristics were measured using a Keithley 2425 source-measurement unit with a filtered Xe lamp, providing the AM $1.5 \mathrm{G}$ solar spectrum. The intensity of the lamp was adjusted to $100 \mathrm{mWcm}^{-2}$.

OFETs were characterized using a semiconductor parameter analyzer (Keithley 4200). Mobilities were obtained from saturation regime curves using standard OFET models.

To further substantiate our hypothesis that molecular packing affects device performance Table S1.

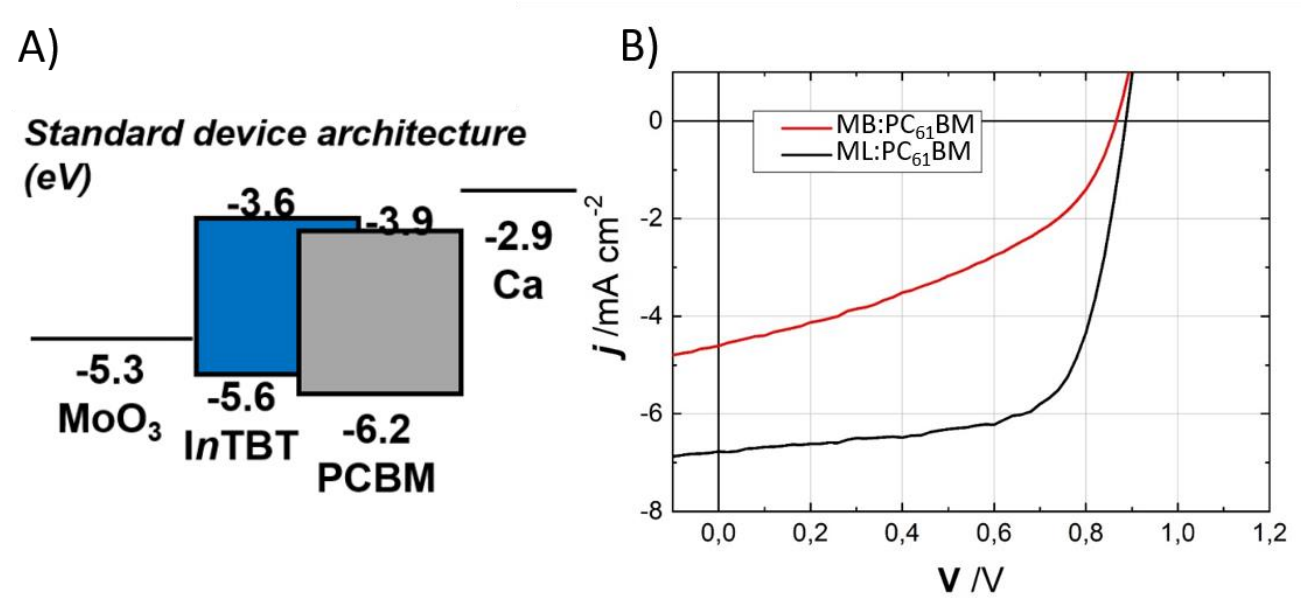

Figure S5 A) Standard solar cell architecture used for device characterization B)Voltamperic characteristics of $M L$ and $M B$ based devices with $P C_{61} B M$ as the electron acceptor.

Bulk heterojunction solar cells with the same thickness of both compounds were made in a standard configuration of $\mathrm{ITO} / \mathrm{MoO}_{3} / \mathrm{BHJ} / \mathrm{Ca} / \mathrm{Ag}$. The PCE of $\mathrm{ML}$ and $\mathrm{MB}$ under $\mathrm{AM} 1.5$ illumination are $4.1 \%$ and $1.7 \%$, respectively. Having a closer look at the short circuit current $\left(6.77 \mathrm{vs.} 4.61 \mathrm{~mA} / \mathrm{cm}^{2}\right)$ and fill factor $(0.68$ vs. 0.42)) (sup info) it is apparent that ML has a higher photogeneration yield and fill factor. A higher charge generation is attributed to less pronounced CT formation among the merocyanines in ML in the presence of $\mathrm{PC}_{61} \mathrm{BM}$ as suggested by theoretical modelling. That a higher fraction of $\mathrm{H}$-type aggregation in $\mathrm{ML}$ improves charge transport is reflected in the higher FF of these devices.

\begin{tabular}{|c|c|c|c|c|c|}
\hline Material & $\mathbf{V}_{\text {oc }} / \mathbf{V}$ & $\mathbf{j} / \mathbf{c m}^{2} / \mathbf{V} \mathbf{s}$ & $\mathbf{F F}$ & $\mathbf{P C E} / \mathbf{\%}$ & $\begin{array}{c}\boldsymbol{\mu} / \mathbf{c m}^{2} \mathbf{V}^{-1} \mathbf{s}^{-1} \\
\mathbf{S C L C}\end{array}$ \\
\hline ML:PC ${ }_{61} \mathbf{B M}$ & 0.87 & 6.77 & 0.68 & 4.09 & $1.5 \cdot 10^{-5}$ \\
& \pm 0.009 & \pm 0.28 & \pm 0.013 & \pm 0.13 & $\pm 0.5 \cdot 10^{-5}$ \\
\hline MB:PC $61 \mathbf{B M}$ & 0.87 & 4.61 & 0.42 & 1.66 & $1.4 \cdot 10^{-7}$ \\
& \pm 0.01 & \pm 0.20 & \pm 0.01 & \pm 0.07 & $\pm 0.5 \cdot 10^{-7}$ \\
\hline
\end{tabular}

Table S1. Device characteristics of merocyanine bulk heterojunction solar cells together with mobility. 


\section{Linear absorption as the function of $\mathrm{PC}_{61} \mathrm{BM}$ content}

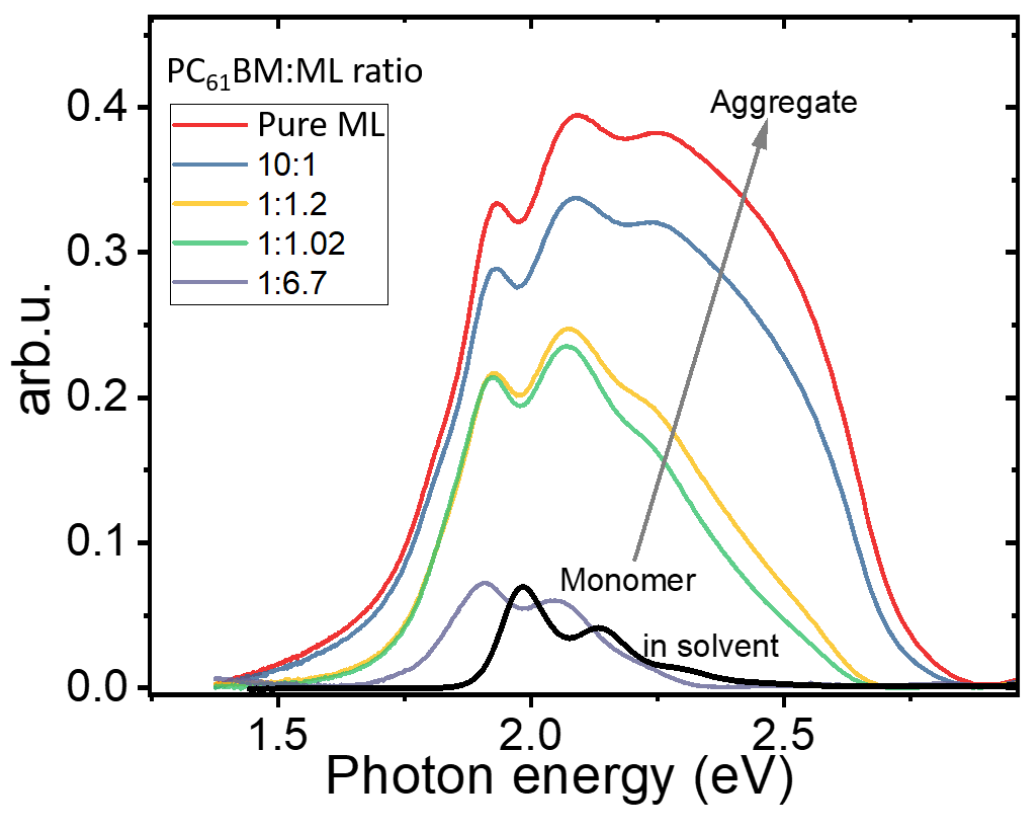

Figure S6 Absorption spectra of $M L: P C_{61} B M$ films with varying $P C_{61} B M: M L$ ratio. The absorption spectrum of $M L$, molecule, dissolved in Acetone solvent is shown in black. With increasing $P_{6}{ }_{61} B M$ ratio, a reduction in spectra weights at the blue side of the spectrum was observed, hint to a reduction in H-aggregate formation. The red shift of the spectrum was attributed to the difference in local surrounding and change in dielectric constant.

\section{DFT calculations}

TDDFT calculations using TD- $\omega$ B97X-D3/6-31G* merocyanine molecules and clusters is shown below. The represented Absorption spectrum was calculated by convoluting excited states with Lorentzian function. 


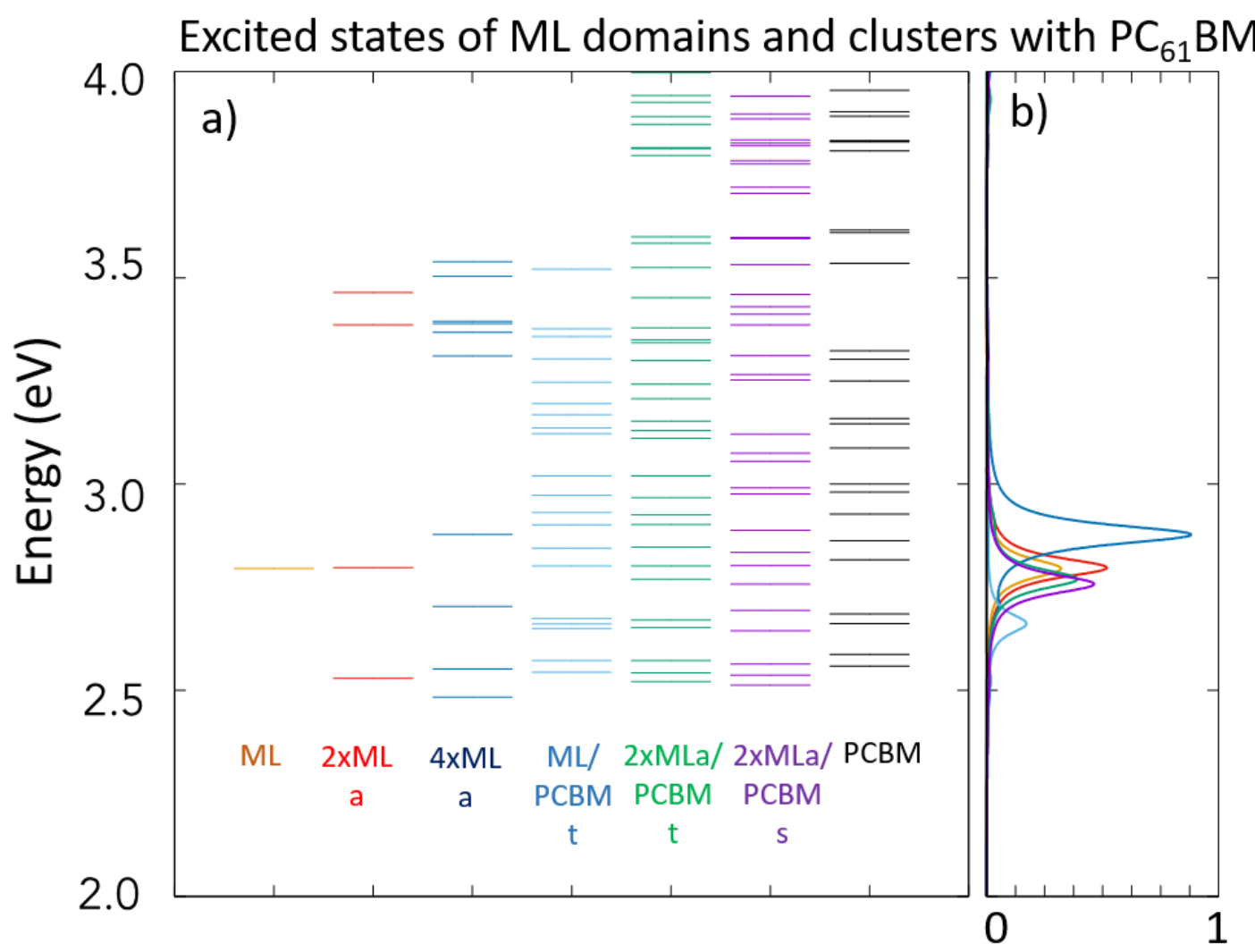

Figure S7 a)Computed TD-wB97X-D3/6-31G* excited state vertical energies for ML molecules and cluster with $P C_{61} B M$; b) Absorption spectra of computed transitions, plotted using the Lorentz convolution of calculated states. 2xML a: dimer with antiparallel alignment, 4XML a: cluster of 4 antiparallel molecules, ML/PCBM t: merocyanine and $P C_{61} B M$ cluster with PC ${ }_{61} B M$ on top, 2x MLa/PCBM: two ML molecules aligned antiparallel with PC61BM on top, $2 x M L a / P C B M s$ :two ML molecules with antiparallel alignment with $P C_{61} B M$ on the side, PCBM: spectrum of the pure $P C_{61} B M$. 


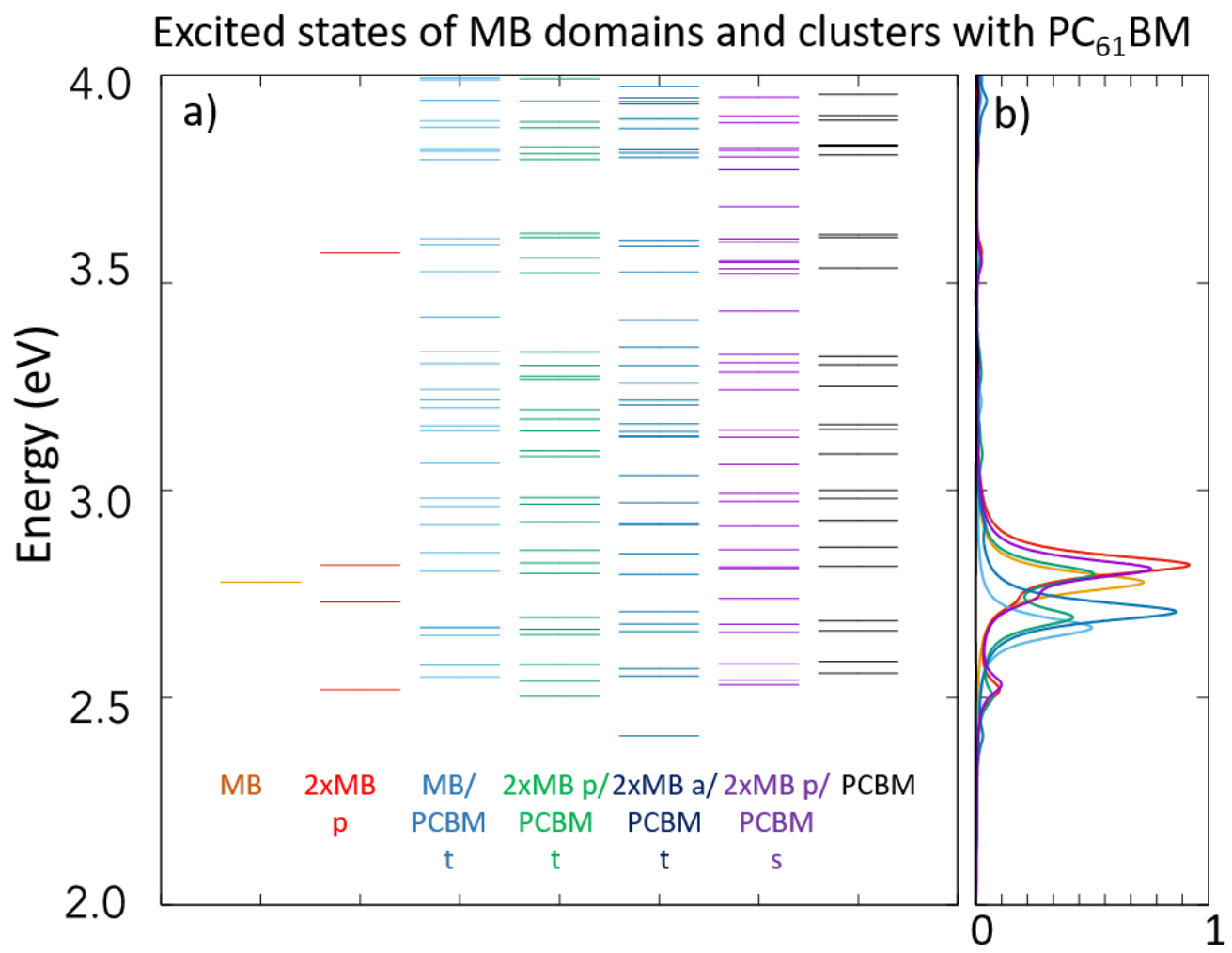

Figure S8 a)Computed TD- $\omega B 97 X-D 3 / 6-31 G^{*}$ excited state vertical energies for MB molecules and cluster with PC61BM; b) Absorption spectra of computed transitions, plotted using the Lorentz convolution of calculated states. $2 x M B$ : dimer with parallel alignment, MB/PCBM t: MB molecule and $P C_{61} B M$ cluster with $P C_{61} B M$ on top, $2 x M B$ p/PCBM: two MB molecules aligned parallel with PC ${ }_{61} B M$ on top, $2 x M B$ a/PCBM $t$ :two MB molecules with antiparallel alignment with $P C_{61} B M$ on the top, $2 x M B$ p/PCBM s :two MB molecules with parallel alignment with $P C_{61} B M$ on the side, $P C B M$ : spectrum of the pure $P C_{61} B M$.

Here below we reported the Cartesian coordinates of the optimized DFT structures (single molecules and structures) considered in the manuscript. The first entry in the data is Atom and three numbers represent $\mathrm{X}, \mathrm{Y}$, $\mathrm{Z}$ coordinates in Angstrom.

\begin{tabular}{lrrr}
\multicolumn{4}{c}{ MB single molecule } \\
C & 5.125662 & 0.030095 & -1.175431 \\
C & 3.841426 & -0.449128 & -0.958693 \\
C & 3.597956 & -1.774943 & -0.616295 \\
C & 4.648618 & -2.663862 & -0.486391 \\
C & 5.951304 & -2.205923 & -0.702365 \\
C & 6.179965 & -0.875320 & -1.042602 \\
C & 2.106775 & -1.983738 & -0.451663 \\
C & 1.567473 & -0.570564 & -0.727906 \\
N & 2.624454 & 0.245273 & -1.031597 \\
C & 0.265629 & -0.142643 & -0.691705 \\
C & -0.865020 & -0.943877 & -0.390085 \\
C & -2.151297 & -0.493418 & -0.317366 \\
S & -2.578489 & 1.204300 & -0.588079
\end{tabular}

$\begin{array}{rrrr}\mathrm{C} & -4.260452 & 0.797220 & -0.290565 \\ \mathrm{~N} & -4.468422 & -0.523975 & -0.010126 \\ \mathrm{C} & -3.368785 & -1.227526 & -0.017698 \\ \mathrm{C} & -5.281062 & 1.716623 & -0.332302 \\ \mathrm{C} & -6.626777 & 1.318967 & -0.078922 \\ \mathrm{~N} & -7.728408 & 1.016408 & 0.124398 \\ \mathrm{C} & -3.459532 & -2.720632 & 0.285647 \\ \mathrm{C} & -2.948235 & -3.541072 & -0.915773 \\ \mathrm{C} & 1.601683 & -2.994783 & -1.498668 \\ \mathrm{C} & 1.796664 & -2.452968 & 0.981073 \\ \mathrm{C} & 2.573241 & 1.663558 & -1.344828 \\ \mathrm{C} & 2.938892 & 2.606393 & -0.183803 \\ \mathrm{C} & -2.655383 & -3.049471 & 1.559729 \\ \mathrm{C} & -4.925967 & -3.106345 & 0.535360\end{array}$




\begin{tabular}{|c|c|c|c|}
\hline C & -5.006824 & 3.081999 & -0.628235 \\
\hline $\mathrm{N}$ & -4.735580 & 4.184611 & -0.873273 \\
\hline $\mathrm{H}$ & -0.710251 & -1.995743 & -0.197272 \\
\hline $\mathrm{H}$ & -2.779215 & -4.110177 & 1.802937 \\
\hline $\mathrm{H}$ & -1.584771 & -2.854337 & 1.456393 \\
\hline $\mathrm{H}$ & -3.021446 & -2.462459 & 2.408206 \\
\hline $\mathrm{H}$ & -4.981674 & -4.178596 & 0.753612 \\
\hline $\mathrm{H}$ & -5.346476 & -2.554287 & 1.379273 \\
\hline $\mathrm{H}$ & -5.548962 & -2.893337 & -0.336744 \\
\hline $\mathrm{H}$ & -3.087167 & -4.607920 & -0.710644 \\
\hline $\mathrm{H}$ & -3.513966 & -3.293952 & -1.819855 \\
\hline $\mathrm{H}$ & -1.888667 & -3.379295 & -1.130495 \\
\hline $\mathrm{H}$ & 0.071695 & 0.903732 & -0.900621 \\
\hline $\mathrm{H}$ & 5.316131 & 1.066171 & -1.433717 \\
\hline $\mathrm{H}$ & 7.195850 & -0.529232 & -1.205540 \\
\hline $\mathrm{H}$ & 6.787792 & -2.889717 & -0.602414 \\
\hline $\mathrm{H}$ & 4.468406 & -3.702238 & -0.220808 \\
\hline $\mathrm{H}$ & 2.320028 & -3.394060 & 1.178369 \\
\hline $\mathrm{H}$ & 2.133317 & -1.715183 & 1.713845 \\
\hline $\mathrm{H}$ & 0.728959 & -2.624154 & 1.138227 \\
\hline $\mathrm{H}$ & 2.100280 & -3.957584 & -1.347309 \\
\hline $\mathrm{H}$ & 0.523158 & -3.157790 & -1.424325 \\
\hline $\mathrm{H}$ & 1.825982 & -2.649455 & -2.511844 \\
\hline $\mathrm{H}$ & 1.571811 & 1.900485 & -1.713095 \\
\hline $\mathrm{H}$ & 3.254469 & 1.832377 & -2.187152 \\
\hline $\mathrm{C}$ & 2.781749 & 4.053011 & -0.681282 \\
\hline $\mathrm{C}$ & 3.492623 & 5.083259 & 0.194558 \\
\hline $\mathrm{H}$ & 3.342610 & 6.094809 & -0.194643 \\
\hline $\mathrm{H}$ & 3.122572 & 5.069155 & 1.224992 \\
\hline $\mathrm{H}$ & 4.571105 & 4.890881 & 0.225384 \\
\hline $\mathrm{H}$ & 3.175417 & 4.131878 & -1.703788 \\
\hline $\mathrm{H}$ & 1.711336 & 4.291975 & -0.745307 \\
\hline $\mathrm{C}$ & 2.108608 & 2.329004 & 1.084067 \\
\hline $\mathrm{C}$ & 2.737742 & 1.318158 & 2.046841 \\
\hline $\mathrm{C}$ & 1.768891 & 0.863957 & 3.137903 \\
\hline $\mathrm{C}$ & 2.428976 & -0.028298 & 4.187236 \\
\hline $\mathrm{H}$ & 1.705875 & -0.368264 & 4.935358 \\
\hline $\mathrm{H}$ & 2.880472 & -0.916945 & 3.729615 \\
\hline $\mathrm{H}$ & 3.227397 & 0.509098 & 4.711314 \\
\hline $\mathrm{H}$ & 1.953894 & 3.266642 & 1.631245 \\
\hline $\mathrm{H}$ & 1.104628 & 1.990995 & 0.798980 \\
\hline $\mathrm{H}$ & 3.627821 & 1.771105 & 2.505910 \\
\hline $\mathrm{H}$ & 3.101988 & 0.439376 & 1.500220 \\
\hline $\mathrm{H}$ & 1.333800 & 1.744439 & 3.628822 \\
\hline $\mathrm{H}$ & 0.930365 & 0.332409 & 2.666857 \\
\hline $\mathrm{H}$ & 3.999690 & 2.447754 & 0.060449 \\
\hline
\end{tabular}

ML single molecule

\begin{tabular}{|c|c|c|c|}
\hline $\mathrm{C}$ & 2.102622 & -3.598767 & \\
\hline S & 1.063195 & -2.208840 & -0.586617 \\
\hline C & 2.395583 & -1.154851 & -0.084762 \\
\hline C & 3.538470 & -1.990133 & 0.243671 \\
\hline $\mathrm{N}$ & 3.358567 & -3.276184 & 0.110188 \\
\hline c & 2.275682 & 0.203763 & -0.041903 \\
\hline $\mathrm{C}$ & 1.114216 & 0.935198 & -0.399237 \\
\hline C & 4.901122 & -1.492444 & 0.718317 \\
\hline $\mathrm{C}$ & 4.754049 & -0.736104 & 2.054082 \\
\hline $\mathrm{C}$ & 1.697708 & -4.896299 & -0.524184 \\
\hline $\mathrm{C}$ & 0.375212 & -5.173837 & -0.972593 \\
\hline $\mathrm{N}$ & -0.713160 & -5.350910 & -1.337933 \\
\hline $\mathrm{C}$ & 5.833666 & -2.691580 & 0.950852 \\
\hline $\mathrm{C}$ & 5.540712 & -0.591167 & -0.357239 \\
\hline C & 2.591925 & -5.981451 & -0.287378 \\
\hline $\mathrm{N}$ & 3.303594 & -6.878690 & -0.100371 \\
\hline $\mathrm{H}$ & 3.143982 & 0.756124 & 0.288926 \\
\hline $\mathrm{H}$ & 5.746082 & -0.447894 & 2.417889 \\
\hline $\mathrm{H}$ & 4.153717 & 0.173667 & 1.971285 \\
\hline $\mathrm{H}$ & 4.288931 & -1.376412 & 2.810485 \\
\hline $\mathrm{H}$ & 6.808975 & -2.325786 & 1.290538 \\
\hline $\mathrm{H}$ & 5.431078 & -3.370318 & 1.706611 \\
\hline $\mathrm{H}$ & 5.975931 & -3.270466 & 0.035111 \\
\hline $\mathrm{H}$ & 6.543802 & -0.295356 & -0.031802 \\
\hline $\mathrm{H}$ & 5.637997 & -1.131617 & -1.304323 \\
\hline $\mathrm{H}$ & 4.970704 & 0.321273 & -0.551137 \\
\hline $\mathrm{C}$ & 0.970515 & 2.296600 & -0.323257 \\
\hline $\mathrm{C}$ & 1.961914 & 3.340718 & 0.211535 \\
\hline $\mathrm{N}$ & -0.164829 & 2.942687 & -0.726940 \\
\hline $\mathrm{C}$ & -0.093753 & 4.318643 & -0.462908 \\
\hline $\mathrm{C}$ & 1.153120 & 4.616318 & 0.079511 \\
\hline $\mathrm{H}$ & 0.267430 & 0.359281 & -0.759254 \\
\hline $\mathrm{C}$ & 3.229483 & 3.423855 & -0.659376 \\
\hline $\mathrm{C}$ & 2.307643 & 3.091524 & 1.690886 \\
\hline $\mathrm{C}$ & 1.473390 & 5.919452 & 0.410674 \\
\hline $\mathrm{C}$ & 0.524157 & 6.923596 & 0.199073 \\
\hline $\mathrm{C}$ & -0.723157 & 6.608660 & -0.333020 \\
\hline C & -1.056089 & 5.295698 & -0.672619 \\
\hline $\mathrm{H}$ & -2.035675 & 5.062049 & -1.076014 \\
\hline $\mathrm{H}$ & -1.456213 & 7.394551 & -0.485929 \\
\hline $\mathrm{H}$ & 0.758670 & 7.951424 & 0.455594 \\
\hline $\mathrm{H}$ & 2.445137 & 6.162571 & 0.832468 \\
\hline $\mathrm{H}$ & 2.910919 & 3.922111 & 2.071235 \\
\hline $\mathrm{H}$ & 1.399068 & 3.023902 & 2.295817 \\
\hline $\mathrm{H}$ & 2.878420 & 2.169517 & 1.828792 \\
\hline $\mathrm{H}$ & 3.847079 & 4.264346 & -0.326758 \\
\hline
\end{tabular}




$\begin{array}{rrrr}\text { H } & 3.833969 & 2.515197 & -0.591541 \\ \text { H } & 2.970024 & 3.585089 & -1.709476 \\ \text { C } & -1.342597 & 2.290780 & -1.270198 \\ \text { C } & -2.277055 & 1.752845 & -0.186019 \\ \text { C } & -3.469941 & 1.002265 & -0.775324 \\ \text { C } & -4.406967 & 0.443888 & 0.295392 \\ \text { C } & -5.595067 & -0.320627 & -0.287502 \\ \text { C } & -6.528019 & -0.886985 & 0.782535 \\ \text { C } & -7.715453 & -1.654226 & 0.201714 \\ \text { C } & -8.648075 & -2.224064 & 1.270909 \\ \text { C } & -9.831373 & -2.988800 & 0.680574 \\ \text { H } & -10.482960 & -3.386231 & 1.465588 \\ \text { H } & -10.437593 & -2.339692 & 0.038021 \\ \text { H } & -9.488401 & -3.832336 & 0.070574 \\ \text { H } & -1.013873 & 1.486536 & -1.936712 \\ \text { H } & -1.862636 & 3.016365 & -1.902865 \\ \text { H } & -1.710230 & 1.087163 & 0.476877 \\ \text { H } & -2.623454 & 2.590788 & 0.432608 \\ \text { H } & -4.033787 & 1.670836 & -1.441462 \\ \text { H } & -3.106157 & 0.176667 & -1.402908 \\ \text { H } & -3.838882 & -0.220699 & 0.960891 \\ \text { H } & -4.774672 & 1.267850 & 0.923178 \\ \text { H } & -6.164920 & 0.343718 & -0.952799 \\ \text { H } & -5.224297 & -1.141560 & -0.916870 \\ \text { H } & -6.898477 & -0.066574 & 1.413889 \\ \text { H } & -5.956749 & -1.550936 & 1.446468 \\ \text { H } & -8.287896 & -0.991026 & -0.462782 \\ \text { H } & -7.344477 & -2.473881 & -0.429747 \\ \text { H } & -9.017454 & -1.404705 & 1.902301 \\ \text { H } & -8.075290 & -2.887218 & 1.932714 \\ & & & \end{array}$

2xMB cluster

$\begin{array}{rrrr}\mathrm{C} & -2.835017 & 1.532596 & 2.613375 \\ \mathrm{C} & -1.668950 & 1.757407 & 1.892146 \\ \mathrm{C} & -1.415436 & 2.974445 & 1.262198 \\ \mathrm{C} & -2.344700 & 3.995817 & 1.328432 \\ \mathrm{C} & -3.527263 & 3.792603 & 2.048494 \\ \mathrm{C} & -3.760468 & 2.575957 & 2.684896 \\ \mathrm{C} & -0.047560 & 2.924502 & 0.609002 \\ \mathrm{C} & 0.397766 & 1.492828 & 0.944553 \\ \mathrm{~N} & -0.597883 & 0.888086 & 1.677666 \\ \mathrm{C} & 1.571290 & 0.862326 & 0.651708 \\ \mathrm{C} & 2.741374 & 1.428678 & 0.071666 \\ \mathrm{C} & 3.928312 & 0.764670 & -0.016160 \\ \mathrm{~S} & 4.085477 & -0.923373 & 0.489241 \\ \mathrm{C} & 5.792726 & -0.855326 & 0.099374 \\ \mathrm{~N} & 6.213544 & 0.369697 & -0.331788\end{array}$

\begin{tabular}{|c|c|c|c|}
\hline & & & \\
\hline & 6.625405 & -1.940174 & \\
\hline & & & \\
\hline & & & \\
\hline & 5.576460 & 98 & \\
\hline & 10 & & \\
\hline & & & \\
\hline & 0.86 & & \\
\hline & -0.59 & & \\
\hline & -0.15 & & \\
\hline & 5.288634 & & \\
\hline & 7.068571 & & \\
\hline & 6.09 & -3.1 & \\
\hline & 5.595466 & -4.1 & \\
\hline & & & \\
\hline & 5.58 & & \\
\hline & & & \\
\hline & 5.86 & & \\
\hline & 7.29 & & \\
\hline & 7.70 & & \\
\hline & 7.32 & & \\
\hline & 5.06 & & \\
\hline & 4.956255 & 329 & \\
\hline & 3.68 & & \\
\hline & $1.66 \mathrm{C}$ & -0.1 & \\
\hline & -3.02 & & \\
\hline & -4.67 & & \\
\hline & -4.261093 & & \\
\hline & -2.15 & 4.94 & \\
\hline & & & \\
\hline & 0.955570 & 3.81 & \\
\hline & & & \\
\hline & -0.521487 & 727 & \\
\hline & & & \\
\hline & -0.847389 & 8048 & \\
\hline & 0.030 & -1.08 & \\
\hline & -1.61 & -0.8 & \\
\hline & -0.577800 & -2.00 & \\
\hline & -0.578351 & & \\
\hline & -0.903128 & -3.178706 & \\
\hline & 0.414830 & -2.01 & \\
\hline & -1.262001 & -1.455 & \\
\hline & -1.587456 & -2.226599 & \\
\hline & 0.088661 & -2.748120 & 3.71 \\
\hline & 1.346854 & -0.364 & 3.90 \\
\hline & & & \\
\hline & 3.288093 & 1.279485 & \\
\hline & 3.715908 & 2.744624 & 3.8872 \\
\hline
\end{tabular}

$\begin{array}{llll}\text { C } & 6.625405 & -1.940174 & 0.222047\end{array}$

$\begin{array}{llll}\text { C } & 8.013993 & -1.843908 & -0.084628\end{array}$

\begin{tabular}{llll}
\hline & 9.150172 & -1.790266 & -0.313265
\end{tabular}

$\begin{array}{ll}2.655598 & -0.875667\end{array}$

$\begin{array}{llll}\text { C } & -0.152894 & 3.150222 & -0.908364\end{array}$

$\begin{array}{llll}\text { C } & -0.159934 & -0.599632 & 3.696265\end{array}$

$\begin{array}{llll}\text { C } & 5.288634 & 3.642893 & 0.274199\end{array}$

$\begin{array}{llll}\text { C } & 7.068571 & 2.743781 & -1.229629\end{array}$

$2.449626-0.284360$

$\begin{array}{llll}\mathrm{H} & 5.587384 & 4.651449 & -0.031019\end{array}$

$\begin{array}{llll}\mathrm{H} & 4.231833 & 3.676489 & 0.553200\end{array}$

\begin{tabular}{llll}
\hline & 7.297759 & 3.763149 & -1.559559
\end{tabular}

H $\quad 7.700004 \quad 2.501915 \quad-0.371172$

$\begin{array}{llll}\mathrm{H} & 7.324138 & 2.047875 & -2.032598\end{array}$

H $\quad 5.069689 \quad 4.013972 \quad-2.482149$

H $\quad 4.956255 \quad 2.306329 \quad-2.935765$

$\begin{array}{llll}\text { H } & 3.684995 & 3.055226 & -1.962524\end{array}$

$\begin{array}{lllll}\mathrm{H} & 1.669862 & -0.159825 & 0.999567\end{array}$

$\begin{array}{llll}\mathrm{H} & -3.021798 & 0.591747 & 3.120447\end{array}$

$\begin{array}{llll}\mathrm{H} & -4.678967 & 2.427834 & 3.244884\end{array}$

H $\quad 0.438718 \quad 4.976158 \quad 1.106402$

$\begin{array}{llll}\mathrm{H} & 0.955570 & 3.812638 & 2.338662\end{array}$

H $\quad 1.868880 \quad 3.977018 \quad 0.827694$

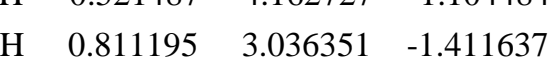

$\begin{array}{llll}\mathrm{H} & -0.847389 & 2.438048 & -1.362836\end{array}$

$\begin{array}{llll}\mathrm{H} & 0.030937 & -1.086009 & 1.584267\end{array}$

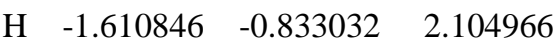

4.173499

$\begin{array}{llll}\text { C } & -0.578351 & -2.171322 & 5.692130\end{array}$

$\mathrm{H} \quad-0.903128 \quad-3.178706 \quad 5.970454$

$\mathrm{H} \quad 0.414830 \quad-2.016883 \quad 6.124756$

$\mathrm{H} \quad-1.262001 \quad-1.455260 \quad 6.162330$

$\mathrm{H} \quad-1.587456 \quad-2.226599 \quad 3.802954$

$\begin{array}{llll}\text { H } & 0.088661 & -2.748120 & 3.716598\end{array}$

$\begin{array}{llll}\text { C } & 1.346854 & -0.364468 & 3.909362\end{array}$

$\begin{array}{llll}\text { C } & 1.776668 & 1.099879 & 4.034239\end{array}$

$\begin{array}{llll}\text { C } & 3.715908 & 2.744624 & 3.887298\end{array}$ 


\begin{tabular}{|c|c|c|c|}
\hline & & & \\
\hline & & & \\
\hline & & & \\
\hline & & & \\
\hline & & & \\
\hline & 1.44 & & \\
\hline & & & \\
\hline & 3.799560 & & \\
\hline & & & \\
\hline & & & \\
\hline & 0 & & \\
\hline & 27 & & \\
\hline & -5.87 & -1. & \\
\hline & -6.96 & & \\
\hline & 17 & -1.5 & \\
\hline & -8.19 & & \\
\hline & & & \\
\hline & 0 & -0.9 & \\
\hline & 57 & & \\
\hline & -2.5 & & \\
\hline & -1.5 & & \\
\hline & & & \\
\hline & -0.10 & -0.2 & \\
\hline & 03 & & \\
\hline & & -1.5 & \\
\hline & 0.82 & & \\
\hline & 2.50 & 77 & \\
\hline & 3.86 & & \\
\hline & & & \\
\hline & 0.99 & -3.8 & \\
\hline & & & \\
\hline & -3.7 & & \\
\hline & & & \\
\hline & -4.4 & & \\
\hline & & & \\
\hline & -0.05 & -4.8 & \\
\hline & & & \\
\hline & & & \\
\hline & 1.77 & & \\
\hline & & & \\
\hline & & & \\
\hline & & & \\
\hline & & -4.963852 & \\
\hline & 2.5023 & & \\
\hline & & & \\
\hline & & & \\
\hline & & & \\
\hline & 1.605609 & -2.947441 & 1.2270 \\
\hline
\end{tabular}

$\begin{array}{lrrr}\mathrm{H} & -0.120004 & -3.371283 & 1.183879 \\ \mathrm{H} & -2.313916 & 0.089168 & -1.074154 \\ \mathrm{H} & -7.141016 & 1.466874 & 0.349084 \\ \mathrm{H} & -9.109033 & 0.354830 & 1.367590 \\ \mathrm{H} & -9.013766 & -2.019480 & 2.040142 \\ \mathrm{H} & -6.928406 & -3.333809 & 1.709701 \\ \mathrm{H} & -5.133480 & -4.189905 & -0.041456 \\ \mathrm{H} & -5.080308 & -3.036465 & -1.385646 \\ \mathrm{H} & -3.576450 & -3.724927 & -0.750649 \\ \mathrm{H} & -4.359745 & -3.467265 & 2.297650 \\ \mathrm{H} & -2.774484 & -3.005168 & 1.663978 \\ \mathrm{H} & -3.753492 & -1.825109 & 2.559184 \\ \mathrm{H} & -3.376631 & 1.644889 & -0.331057 \\ \mathrm{H} & -4.941746 & 2.073715 & 0.292780 \\ \mathrm{C} & -4.714876 & 3.392914 & -1.949618 \\ \mathrm{C} & -5.468472 & 4.051443 & -3.103778 \\ \mathrm{H} & -5.309540 & 5.134236 & -3.101917 \\ \mathrm{H} & -5.142386 & 3.675572 & -4.078514 \\ \mathrm{H} & -6.546095 & 3.870323 & -3.019573 \\ \mathrm{H} & -5.040756 & 3.857442 & -1.009265 \\ \mathrm{H} & -3.639753 & 3.606054 & -2.034176 \\ \mathrm{C} & -4.174641 & 1.139118 & -2.993570 \\ \mathrm{C} & -4.689418 & -0.264959 & -3.322186 \\ \mathrm{C} & -3.749864 & -1.028154 & -4.254550 \\ \mathrm{C} & -4.257772 & -2.426619 & -4.596416 \\ \mathrm{H} & -3.572405 & -2.944809 & -5.274419 \\ \mathrm{H} & -4.356300 & -3.038722 & -3.691913 \\ \mathrm{H} & -5.240954 & -2.384896 & -5.079158 \\ \mathrm{H} & -4.241942 & 1.740797 & -3.907699 \\ \mathrm{H} & -3.101692 & 1.101591 & -2.757621 \\ \mathrm{H} & -5.683909 & -0.180918 & -3.782237 \\ \mathrm{H} & -4.829674 & -0.850078 & -2.407826 \\ \mathrm{H} & -3.601275 & -0.451286 & -5.176871 \\ \mathrm{H} & -2.762775 & -1.104954 & -3.779188 \\ \mathrm{H} & -5.985503 & 1.651919 & -1.939272 \\ & & & \end{array}$

2xML cluster

$\begin{array}{lrrr}\mathrm{C} & 1.447551 & -2.661895 & -1.514304 \\ \mathrm{~S} & 2.500544 & -1.292457 & -1.222984 \\ \mathrm{C} & 1.119795 & -0.214802 & -1.489663 \\ \mathrm{C} & -0.030313 & -1.019853 & -1.809112 \\ \mathrm{~N} & 0.166601 & -2.318701 & -1.818272 \\ \mathrm{C} & 1.233699 & 1.147040 & -1.347508 \\ \mathrm{C} & 2.397410 & 1.792942 & -0.893973 \\ \mathrm{C} & -1.434406 & -0.494460 & -2.089400 \\ \mathrm{C} & -1.428365 & 0.419002 & -3.330793 \\ \mathrm{C} & 1.871571 & -3.970929 & -1.401301\end{array}$




\begin{tabular}{|c|c|c|c|}
\hline C & 3.222117 & -4.253885 & -1.061077 \\
\hline $\mathrm{N}$ & 4.333030 & -4.435039 & -0.771309 \\
\hline $\mathrm{C}$ & -2.379887 & -1.673983 & -2.364158 \\
\hline $\mathrm{C}$ & -1.958434 & 0.261499 & -0.853068 \\
\hline $\mathrm{C}$ & 0.949984 & -5.047295 & -1.540649 \\
\hline $\mathrm{N}$ & 0.203986 & -5.934477 & -1.611095 \\
\hline $\mathrm{H}$ & 0.362256 & 1.743775 & -1.580898 \\
\hline $\mathrm{H}$ & -2.452901 & 0.738565 & -3.549265 \\
\hline $\mathrm{H}$ & -0.822803 & 1.319734 & -3.198533 \\
\hline $\mathrm{H}$ & -1.046050 & -0.117634 & -4.205110 \\
\hline $\mathrm{H}$ & -3.389015 & -1.289773 & -2.550443 \\
\hline $\mathrm{H}$ & -2.057393 & -2.252795 & -3.233259 \\
\hline $\mathrm{H}$ & -2.420342 & -2.358819 & -1.512917 \\
\hline $\mathrm{H}$ & -2.993509 & 0.576767 & -1.024848 \\
\hline $\mathrm{H}$ & -1.942084 & -0.389374 & 0.027016 \\
\hline $\mathrm{H}$ & -1.379846 & 1.156128 & -0.609218 \\
\hline $\mathrm{C}$ & 2.598838 & 3.154085 & -0.791475 \\
\hline $\mathrm{C}$ & 1.666712 & 4.299807 & -1.186907 \\
\hline $\mathrm{N}$ & 3.750375 & 3.668212 & -0.291728 \\
\hline C & 3.731039 & 5.077496 & -0.306744 \\
\hline $\mathrm{C}$ & 2.509542 & 5.503884 & -0.818294 \\
\hline $\mathrm{H}$ & 3.212048 & 1.147771 & -0.586211 \\
\hline $\mathrm{C}$ & 0.370422 & 4.286379 & -0.358069 \\
\hline $\mathrm{C}$ & 1.374331 & 4.286122 & -2.698174 \\
\hline $\mathrm{C}$ & 2.229565 & 6.854645 & -0.908305 \\
\hline $\mathrm{C}$ & 3.198107 & 7.771154 & -0.492928 \\
\hline $\mathrm{C}$ & 4.422424 & 7.328270 & 0.000721 \\
\hline $\mathrm{C}$ & 4.711919 & 5.966550 & 0.102922 \\
\hline $\mathrm{H}$ & 5.670051 & 5.634624 & 0.488413 \\
\hline $\mathrm{H}$ & 5.167282 & 8.051121 & 0.318130 \\
\hline $\mathrm{H}$ & 2.992130 & 8.834694 & -0.549861 \\
\hline $\mathrm{H}$ & 1.270586 & 7.200243 & -1.283098 \\
\hline $\mathrm{H}$ & 0.790047 & 5.172420 & -2.964248 \\
\hline $\mathrm{H}$ & 2.301511 & 4.299037 & -3.278696 \\
\hline $\mathrm{H}$ & 0.800168 & 3.400943 & -2.987892 \\
\hline $\mathrm{H}$ & -0.189295 & 5.208291 & -0.540378 \\
\hline $\mathrm{H}$ & -0.276586 & 3.443449 & -0.619486 \\
\hline $\mathrm{H}$ & 0.589328 & 4.233554 & 0.710910 \\
\hline $\mathrm{C}$ & 4.817905 & 2.848977 & 0.261128 \\
\hline C & 5.610624 & 2.060694 & -0.797984 \\
\hline $\mathrm{C}$ & 6.037329 & 0.684593 & -0.279958 \\
\hline $\mathrm{C}$ & 6.536974 & -0.253051 & -1.377348 \\
\hline $\mathrm{C}$ & 6.926377 & -1.631847 & -0.843900 \\
\hline $\mathrm{C}$ & 7.254722 & -2.636052 & -1.947480 \\
\hline $\mathrm{C}$ & 7.742627 & -3.978065 & -1.404230 \\
\hline C & 7.947400 & -5.027671 & -2.495900 \\
\hline $\mathrm{C}$ & 8.447148 & -6.362473 & -1.946537 \\
\hline $\mathrm{H}$ & 8.579550 & -7.101271 & -2.744168 \\
\hline
\end{tabular}

\begin{tabular}{|c|c|c|c|}
\hline & & & \\
\hline & & & \\
\hline & & & \\
\hline & & & \\
\hline & & & \\
\hline & & & \\
\hline & & & \\
\hline & & & \\
\hline & & & \\
\hline & 51 & & \\
\hline & 7.78 & & \\
\hline & & & \\
\hline & & & \\
\hline & & & \\
\hline & & & \\
\hline & 8 & & \\
\hline & 8.65 & & \\
\hline & 6.95 & & \\
\hline & -1.6 & & \\
\hline & -2.6 & & \\
\hline & 0 & & \\
\hline & -0.0 & & \\
\hline & -0.3 & & \\
\hline & -1.22 & & \\
\hline & -2.3 & & \\
\hline & & & \\
\hline & 8 & & \\
\hline & & & \\
\hline & -3.4 & & \\
\hline & -4.5 & & \\
\hline & & & \\
\hline & & & \\
\hline & -1.24 & & \\
\hline & -0.54 & & \\
\hline & & & \\
\hline & 2.98 & & \\
\hline & & & \\
\hline & 6 & & \\
\hline & & & \\
\hline & & & \\
\hline & & & \\
\hline & & & \\
\hline & 1.077336 & & \\
\hline & & & \\
\hline & -2.388308 & -2.6493 & \\
\hline & & -3.6283 & \\
\hline & & & \\
\hline & -3.248368 & -4.709500 & $1.871 ?$ \\
\hline
\end{tabular}




\begin{tabular}{|c|c|c|c|}
\hline C & -1.894557 & -4.938002 & 2.088318 \\
\hline $\mathrm{H}$ & -3.308615 & -0.745560 & 2.362041 \\
\hline C & -0.572023 & -3.680503 & 3.820781 \\
\hline C & -0.173650 & -3.316429 & 1.327922 \\
\hline$C$ & -1.371097 & -6.207666 & 1.930384 \\
\hline $\mathrm{C}$ & -2.228224 & -7.245956 & 1.559295 \\
\hline $\mathrm{C}$ & -3.582218 & -7.001129 & 1.347429 \\
\hline $\mathrm{C}$ & -4.118881 & -5.720986 & 1.497083 \\
\hline $\mathrm{H}$ & -5.171825 & -5.539025 & 1.309706 \\
\hline $\mathrm{H}$ & -4.234792 & -7.815009 & 1.047869 \\
\hline $\mathrm{H}$ & -1.831716 & -8.245444 & 1.417372 \\
\hline $\mathrm{H}$ & -0.310455 & -6.395063 & 2.069842 \\
\hline $\mathrm{H}$ & 0.605376 & -4.084652 & 1.335744 \\
\hline $\mathrm{H}$ & -0.640237 & -3.324755 & 0.339509 \\
\hline $\mathrm{H}$ & 0.306793 & -2.344188 & 1.470086 \\
\hline $\mathrm{H}$ & 0.175740 & -4.479109 & 3.847868 \\
\hline $\mathrm{H}$ & -0.070258 & -2.742912 & 4.077639 \\
\hline $\mathrm{H}$ & -1.323661 & -3.888983 & 4.587648 \\
\hline $\mathrm{C}$ & -4.812441 & -2.734679 & 1.800602 \\
\hline $\mathrm{C}$ & -4.890842 & -2.188759 & 0.374319 \\
\hline $\mathrm{C}$ & -6.193357 & -1.445128 & 0.090095 \\
\hline $\mathrm{C}$ & -6.208562 & -0.824172 & -1.306694 \\
\hline $\mathrm{C}$ & -7.495738 & -0.064179 & -1.621647 \\
\hline $\mathrm{C}$ & -7.484489 & 0.577873 & -3.008694 \\
\hline $\mathrm{C}$ & -8.767155 & 1.344163 & -3.329869 \\
\hline $\mathrm{C}$ & -8.753307 & 1.991115 & -4.715132 \\
\hline $\mathrm{C}$ & -10.039036 & 2.754772 & -5.026922 \\
\hline $\mathrm{H}$ & -10.003606 & 3.208476 & -6.022730 \\
\hline $\mathrm{H}$ & -10.909414 & 2.089313 & -4.992256 \\
\hline $\mathrm{H}$ & -10.205381 & 3.556730 & -4.298587 \\
\hline $\mathrm{H}$ & -4.989850 & -1.943824 & 2.536373 \\
\hline $\mathrm{H}$ & -5.581484 & -3.491814 & 1.974815 \\
\hline $\mathrm{H}$ & -4.044946 & -1.511238 & 0.210143 \\
\hline $\mathrm{H}$ & -4.763297 & -3.019021 & -0.331799 \\
\hline $\mathrm{H}$ & -7.046941 & -2.128503 & 0.199484 \\
\hline $\mathrm{H}$ & -6.332828 & -0.651882 & 0.837961 \\
\hline $\mathrm{H}$ & -5.353938 & -0.138778 & -1.400805 \\
\hline $\mathrm{H}$ & -6.053971 & -1.612077 & -2.057513 \\
\hline $\mathrm{H}$ & -8.352169 & -0.748639 & -1.542967 \\
\hline $\mathrm{H}$ & -7.652327 & 0.714519 & -0.862385 \\
\hline $\mathrm{H}$ & -7.325043 & -0.200345 & -3.768801 \\
\hline $\mathrm{H}$ & -6.626175 & 1.260759 & -3.083738 \\
\hline $\mathrm{H}$ & -9.626600 & 0.662407 & -3.256486 \\
\hline $\mathrm{H}$ & -8.926364 & 2.120830 & -2.568592 \\
\hline $\mathrm{H}$ & -8.592910 & 1.214652 & -5.475218 \\
\hline $\mathrm{H}$ & -7.894977 & 2.672479 & -4.786203 \\
\hline
\end{tabular}

2xMB: $\mathrm{PC}_{61} \mathrm{BM}\left(\mathrm{PC}_{61} \mathrm{BM}\right.$ on the side $)$ cluster

$$
\begin{aligned}
& \begin{array}{llll}
\text { C } & -4.389469 & -3.428727 & 2.798151
\end{array} \\
& \begin{array}{llll}
\text { C } & -5.327061 & -2.552211 & 2.266288
\end{array} \\
& \begin{array}{llll}
\text { C } & -6.673006 & -2.895128 & 2.152871
\end{array} \\
& \begin{array}{llll}
\text { C } & -7.106569 & -4.145107 & 2.553784
\end{array} \\
& \begin{array}{llll}
\text { C } & -6.179068 & -5.047101 & 3.086869
\end{array} \\
& \begin{array}{llll}
\text { C } & -4.840038 & -4.684591 & 3.210126
\end{array} \\
& \begin{array}{llll}
\text { C } & -7.445032 & -1.713593 & 1.598494
\end{array} \\
& \begin{array}{llll}
\text { C } & -6.318860 & -0.692598 & 1.375481
\end{array} \\
& \begin{array}{llll}
\mathrm{N} & -5.133310 & -1.254301 & 1.789102
\end{array} \\
& \begin{array}{llll}
\text { C } & -6.393830 & 0.586213 & 0.905684
\end{array} \\
& \begin{array}{llll}
\text { C } & -7.567797 & 1.338855 & 0.623973
\end{array} \\
& \begin{array}{llll}
\text { C } & -7.550721 & 2.668514 & 0.324300
\end{array} \\
& \begin{array}{llll}
\text { S } & -6.033371 & 3.561074 & 0.152000
\end{array} \\
& \text { C } \quad-6.955221 \quad 5.025391 \quad-0.124361 \\
& \begin{array}{llll}
\mathrm{N} & -8.306770 & 4.852119 & -0.044797
\end{array} \\
& \begin{array}{llll}
\text { C } & -8.650704 & 3.613437 & 0.172203
\end{array} \\
& \begin{array}{llll}
\text { C } & -6.362989 & 6.235535 & -0.392228
\end{array} \\
& \begin{array}{llll}
\text { C } & -7.143316 & 7.411488 & -0.590868
\end{array} \\
& \begin{array}{llll}
\mathrm{N} & -7.752458 & 8.387338 & -0.742314
\end{array} \\
& \begin{array}{llll}
\text { C } & -10.130483 & 3.276640 & 0.304975
\end{array} \\
& \begin{array}{llll}
\text { C } & -10.542282 & 2.215732 & -0.732716
\end{array} \\
& \begin{array}{llll}
\text { C } & -8.158910 & -2.086349 & 0.289344
\end{array} \\
& \begin{array}{llll}
\text { C } & -8.454262 & -1.225827 & 2.656781
\end{array} \\
& \begin{array}{llll}
\text { C } & -3.818718 & -0.639041 & 1.767954
\end{array} \\
& \begin{array}{llll}
\text { C } & -3.330843 & 0.013324 & 3.076061
\end{array} \\
& \begin{array}{llll}
\text { C } & -10.414272 & 2.782373 & 1.738688
\end{array} \\
& \begin{array}{llll}
\text { C } & -10.965913 & 4.540597 & 0.052199
\end{array} \\
& \begin{array}{llll}
\text { C } & -4.943857 & 6.319142 & -0.472295
\end{array} \\
& \begin{array}{llll}
\mathrm{N} & -3.782759 & 6.322465 & -0.522876
\end{array} \\
& \begin{array}{cccc}
\mathrm{H} & -8.530911 & 0.850203 & 0.686605
\end{array} \\
& \begin{array}{llll}
\mathrm{H} & -11.488436 & 2.602036 & 1.854028
\end{array} \\
& \begin{array}{llll}
\mathrm{H} & -9.890209 & 1.853516 & 1.980592
\end{array} \\
& \begin{array}{llll}
\mathrm{H} & -10.114641 & 3.535616 & 2.475105
\end{array} \\
& \begin{array}{cccc}
\mathrm{H} & -12.028726 & 4.291057 & 0.145758
\end{array} \\
& \begin{array}{llll}
\mathrm{H} & -10.725898 & 5.330596 & 0.768032
\end{array} \\
& \begin{array}{llll}
\mathrm{H} & -10.784286 & 4.937810 & -0.949613
\end{array} \\
& \begin{array}{cccc}
\mathrm{H} & -11.621399 & 2.042308 & -0.662258
\end{array} \\
& \mathrm{H} \quad-10.322274 \quad 2.564926-1.746079 \\
& \begin{array}{llll}
\mathrm{H} & -10.048027 & 1.251248 & -0.595842
\end{array} \\
& \begin{array}{llll}
\mathrm{H} & -5.460030 & 1.132992 & 0.840336
\end{array} \\
& \begin{array}{llll}
\mathrm{H} & -3.347480 & -3.150298 & 2.913372
\end{array} \\
& \begin{array}{cccc}
\mathrm{H} & -4.127244 & -5.387956 & 3.630069
\end{array} \\
& \begin{array}{cccc}
\mathrm{H} & -6.506749 & -6.028977 & 3.413150
\end{array} \\
& \begin{array}{llll}
\mathrm{H} & -8.153675 & -4.422417 & 2.465745
\end{array} \\
& \mathrm{H} \quad-9.164139 \quad-2.028970 \quad 2.879549 \\
& \begin{array}{llll}
\mathrm{H} & -7.948677 & -0.950624 & 3.586713
\end{array}
\end{aligned}
$$




\begin{tabular}{|c|c|c|c|}
\hline $\mathrm{H}$ & -9.025662 & -0.362294 & 2.306973 \\
\hline $\mathrm{H}$ & -8.917231 & -2.850670 & 0.489945 \\
\hline $\mathrm{H}$ & -8.656977 & -1.228846 & -0.170837 \\
\hline $\mathrm{H}$ & -7.452016 & -2.487520 & -0.441753 \\
\hline $\mathrm{H}$ & -3.789509 & 0.085756 & 0.951926 \\
\hline $\mathrm{H}$ & -3.124197 & -1.432071 & 1.479829 \\
\hline $\mathrm{C}$ & -1.820026 & 0.273083 & 2.927573 \\
\hline $\mathrm{C}$ & -1.097936 & 0.565762 & 4.240828 \\
\hline $\mathrm{H}$ & -0.026247 & 0.704260 & 4.068624 \\
\hline $\mathrm{H}$ & -1.470506 & 1.473619 & 4.725171 \\
\hline $\mathrm{H}$ & -1.219651 & -0.264531 & 4.945912 \\
\hline $\mathrm{H}$ & -1.342114 & -0.600451 & 2.465288 \\
\hline $\mathrm{H}$ & -1.667261 & 1.106044 & 2.227477 \\
\hline $\mathrm{C}$ & -4.084640 & 1.309477 & 3.423804 \\
\hline $\mathrm{C}$ & -5.435029 & 1.124477 & 4.121644 \\
\hline $\mathrm{C}$ & -6.277316 & 2.400147 & 4.112651 \\
\hline $\mathrm{C}$ & -7.673658 & 2.196388 & 4.694362 \\
\hline $\mathrm{H}$ & -8.244332 & 3.130577 & 4.696279 \\
\hline $\mathrm{H}$ & -8.236287 & 1.465275 & 4.101555 \\
\hline $\mathrm{H}$ & -7.628501 & 1.827168 & 5.725752 \\
\hline $\mathrm{H}$ & -3.453895 & 1.933411 & 4.067889 \\
\hline $\mathrm{H}$ & -4.219493 & 1.894626 & 2.503177 \\
\hline $\mathrm{H}$ & -5.264960 & 0.793619 & 5.155796 \\
\hline $\mathrm{H}$ & -6.005073 & 0.325880 & 3.638826 \\
\hline $\mathrm{H}$ & -5.752592 & 3.190873 & 4.664799 \\
\hline $\mathrm{H}$ & -6.368558 & 2.757222 & 3.078982 \\
\hline $\mathrm{H}$ & -3.475596 & -0.712661 & 3.890813 \\
\hline $\mathrm{C}$ & 13.159274 & 0.100958 & -1.805789 \\
\hline $\mathrm{C}$ & 12.333317 & 0.569846 & -0.629116 \\
\hline $\mathrm{C}$ & 11.091953 & -0.287232 & -0.415248 \\
\hline $\mathrm{C}$ & 10.261598 & 0.202674 & 0.769579 \\
\hline $\mathrm{C}$ & 8.978210 & -0.601141 & 0.954517 \\
\hline $\mathrm{C}$ & 7.766837 & 0.113743 & 1.500693 \\
\hline $\mathrm{C}$ & 7.860496 & -0.357051 & -0.025338 \\
\hline $\mathrm{C}$ & 6.832599 & -1.343018 & -0.457068 \\
\hline $\mathrm{C}$ & 5.956806 & -1.953020 & 0.411859 \\
\hline $\mathrm{C}$ & 5.869462 & -1.520611 & 1.818767 \\
\hline $\mathrm{C}$ & 6.663119 & -0.499605 & 2.291027 \\
\hline $\mathrm{C}$ & 6.086485 & 0.512827 & 3.133868 \\
\hline $\mathrm{C}$ & 4.760318 & 0.442463 & 3.537810 \\
\hline $\mathrm{C}$ & 3.953538 & 1.646709 & 3.591836 \\
\hline $\mathrm{C}$ & 2.619886 & 1.305592 & 3.142401 \\
\hline C & 1.899159 & 2.203796 & 2.368671 \\
\hline C & 1.131756 & 1.729180 & 1.236017 \\
\hline $\mathrm{C}$ & 1.241402 & 2.714644 & 0.178782 \\
\hline $\mathrm{C}$ & 1.325851 & 2.308072 & -1.142996 \\
\hline $\mathrm{C}$ & 1.308593 & 0.896578 & -1.468830 \\
\hline $\mathrm{C}$ & 1.206312 & -0.048692 & -0.459864 \\
\hline
\end{tabular}

\begin{tabular}{|c|c|c|c|}
\hline C & 1.119075 & 0.377152 & 0.924437 \\
\hline $\mathrm{C}$ & 1.873297 & -0.559113 & 1.729945 \\
\hline $\mathrm{C}$ & 2.608466 & -0.106986 & 2.814747 \\
\hline & 3.934939 & -0.636200 & 3.063433 \\
\hline C & 4.480755 & -1.587056 & 2.208632 \\
\hline C & 3.710324 & -2.056817 & 1.075442 \\
\hline $\mathrm{C}$ & 2.439290 & -1.557301 & 0.842428 \\
\hline $\mathrm{C}$ & 2.020252 & -1.244205 & -0.509889 \\
\hline $\mathrm{C}$ & 2.885737 & -1.455802 & -1.570789 \\
\hline $\mathrm{C}$ & 4.211836 & -1.986918 & -1.326860 \\
\hline $\mathrm{C}$ & 4.621127 & -2.275366 & -0.029698 \\
\hline $\mathrm{C}$ & 5.122761 & -1.330778 & -2.226501 \\
\hline C & 6.394912 & -0.998411 & -1.782017 \\
\hline $\mathrm{C}$ & 6.977520 & 0.277563 & -2.139116 \\
\hline $\mathrm{C}$ & 7.784927 & 0.733052 & -1.038459 \\
\hline $\mathrm{C}$ & 7.786222 & 2.071499 & -0.712361 \\
\hline $\mathrm{C}$ & 7.695870 & 2.504648 & 0.695149 \\
\hline $\mathrm{C}$ & 7.610065 & 1.579474 & 1.711954 \\
\hline C & 6.667475 & 1.789972 & 2.778048 \\
\hline $\mathrm{C}$ & 5.897503 & 2.943070 & 2.838062 \\
\hline $\mathrm{C}$ & 4.508681 & 2.867802 & 3.249493 \\
\hline $\mathrm{C}$ & 3.757562 & 3.808083 & 2.443439 \\
\hline $\mathrm{C}$ & 2.480456 & 3.483831 & 2.012135 \\
\hline $\mathrm{C}$ & 2.073309 & 3.798861 & 0.658603 \\
\hline $\mathrm{C}$ & 2.960088 & 4.422614 & -0.205579 \\
\hline C & 4.293876 & 4.759169 & 0.244284 \\
\hline $\mathrm{C}$ & 4.685264 & 4.460302 & 1.539446 \\
\hline $\mathrm{C}$ & 6.007586 & 3.923120 & 1.790114 \\
\hline C & 6.880364 & 3.695481 & 0.733177 \\
\hline $\mathrm{C}$ & 6.468487 & 4.011234 & -0.619013 \\
\hline $\mathrm{C}$ & 5.208117 & 4.534017 & -0.858991 \\
\hline $\mathrm{C}$ & 4.434874 & 4.070347 & -1.994927 \\
\hline $\mathrm{C}$ & 3.047202 & 3.997193 & -1.589723 \\
\hline $\mathrm{C}$ & 2.243816 & 2.965440 & -2.050346 \\
\hline C & 2.791856 & 1.960578 & -2.938243 \\
\hline $\mathrm{C}$ & 2.211338 & 0.680883 & -2.580200 \\
\hline $\mathrm{C}$ & 2.982917 & -0.470563 & -2.630464 \\
\hline $\mathrm{C}$ & 4.370280 & -0.393105 & -3.038142 \\
\hline $\mathrm{C}$ & 4.925535 & 0.829398 & -3.376432 \\
\hline $\mathrm{C}$ & 4.120786 & 2.032630 & -3.327989 \\
\hline $\mathrm{C}$ & 4.961358 & 3.111353 & -2.845271 \\
\hline $\mathrm{C}$ & 6.284271 & 2.572855 & -2.598840 \\
\hline $\mathrm{C}$ & 6.260469 & 1.170962 & -2.922537 \\
\hline $\mathrm{C}$ & 7.021971 & 3.006982 & -1.504208 \\
\hline $\mathrm{C}$ & 9.204165 & -2.014437 & 1.419727 \\
\hline $\mathrm{C}$ & 9.350309 & -3.057788 & 0.505656 \\
\hline $\mathrm{C}$ & 9.624018 & -4.348357 & 0.948097 \\
\hline $\mathrm{C}$ & 9.751795 & -4.606799 & 2.309545 \\
\hline
\end{tabular}




\begin{tabular}{|c|c|c|c|}
\hline $\mathrm{C}$ & 9.608165 & -3.569527 & 3.227514 \\
\hline $\mathrm{C}$ & 9.336422 & -2.280401 & 2.783147 \\
\hline $\mathrm{C}$ & 15.097402 & 0.507210 & -3.054538 \\
\hline $\mathrm{H}$ & 12.065590 & 1.619766 & -0.802465 \\
\hline $\mathrm{H}$ & 12.976892 & 0.568387 & 0.259354 \\
\hline $\mathrm{H}$ & 10.481142 & -0.268541 & -1.325494 \\
\hline $\mathrm{H}$ & 11.391371 & -1.329431 & -0.266203 \\
\hline $\mathrm{H}$ & 10.025950 & 1.263314 & 0.634698 \\
\hline $\mathrm{H}$ & 10.840460 & 0.128251 & 1.698649 \\
\hline $\mathrm{H}$ & 9.247371 & -2.858659 & -0.557438 \\
\hline $\mathrm{H}$ & 9.736263 & -5.151870 & 0.226691 \\
\hline $\mathrm{H}$ & 9.961774 & -5.614455 & 2.655186 \\
\hline $\mathrm{H}$ & 9.704687 & -3.764771 & 4.291224 \\
\hline $\mathrm{H}$ & 9.217446 & -1.471332 & 3.499488 \\
\hline $\mathrm{H}$ & 15.919874 & 1.221617 & -3.036446 \\
\hline $\mathrm{H}$ & 14.553702 & 0.572929 & -4.000204 \\
\hline H & 15.472471 & -0.511836 & -2.931491 \\
\hline $\mathrm{O}$ & 14.251050 & 0.866245 & -1.962585 \\
\hline $\mathrm{O}$ & 12.889960 & -0.833231 & -2.522930 \\
\hline $\mathrm{C}$ & -2.379964 & -7.128404 & 0.412303 \\
\hline $\mathrm{C}$ & -2.298660 & -5.797046 & 0.030639 \\
\hline $\mathrm{C}$ & -1.079120 & -5.138668 & -0.092713 \\
\hline $\mathrm{C}$ & 0.102414 & -5.802919 & 0.176625 \\
\hline $\mathrm{C}$ & 0.046538 & -7.144919 & 0.564538 \\
\hline $\mathrm{C}$ & -1.180817 & -7.792863 & 0.678745 \\
\hline $\mathrm{C}$ & -1.328022 & -3.701484 & -0.498622 \\
\hline $\mathrm{C}$ & -2.853710 & -3.685770 & -0.638215 \\
\hline $\mathrm{N}$ & -3.340157 & -4.910675 & -0.280244 \\
\hline $\mathrm{C}$ & -3.663138 & -2.672106 & -1.077163 \\
\hline $\mathrm{C}$ & -3.288704 & -1.357115 & -1.449727 \\
\hline $\mathrm{C}$ & -4.170427 & -0.460297 & -1.986615 \\
\hline $\mathrm{S}$ & -5.831549 & -0.934835 & -2.366145 \\
\hline $\mathrm{C}$ & -6.211667 & 0.746634 & -2.665542 \\
\hline $\mathrm{N}$ & -5.131957 & 1.576245 & -2.609232 \\
\hline $\mathrm{C}$ & -4.033854 & 0.959922 & -2.254866 \\
\hline $\mathrm{C}$ & -7.492106 & 1.185754 & -2.910402 \\
\hline $\mathrm{C}$ & -7.774727 & 2.567362 & -3.105744 \\
\hline $\mathrm{N}$ & -8.048457 & 3.687512 & -3.240937 \\
\hline $\mathrm{C}$ & -2.745501 & 1.764952 & -2.148519 \\
\hline C & -2.255442 & 1.786173 & -0.686479 \\
\hline $\mathrm{C}$ & -0.874770 & -2.767513 & 0.636020 \\
\hline $\mathrm{C}$ & -0.614575 & -3.370797 & -1.819268 \\
\hline $\mathrm{C}$ & -4.737399 & -5.290065 & -0.150419 \\
\hline $\mathrm{C}$ & -5.367171 & -6.057962 & -1.325489 \\
\hline $\mathrm{C}$ & -1.683108 & 1.155584 & -3.084435 \\
\hline $\mathrm{C}$ & -3.000737 & 3.215492 & -2.585349 \\
\hline $\mathrm{C}$ & -8.571116 & 0.260986 & -2.864783 \\
\hline N & -9.418568 & -0.531229 & -2.786017 \\
\hline
\end{tabular}

$$
\begin{aligned}
& \begin{array}{llll}
\mathrm{H} & -2.273212 & -1.022575 & -1.274299
\end{array} \\
& \begin{array}{llll}
\mathrm{H} & -0.802397 & 1.804700 & -3.102803
\end{array} \\
& \begin{array}{llll}
\mathrm{H} & -1.353779 & 0.159702 & -2.773536
\end{array} \\
& \begin{array}{llll}
\mathrm{H} & -2.068079 & 1.082310 & -4.106851
\end{array} \\
& \begin{array}{llll}
\mathrm{H} & -2.064156 & 3.778860 & -2.517869
\end{array} \\
& \begin{array}{lllll}
\mathrm{H} & -3.365538 & 3.262791 & -3.614700
\end{array} \\
& \begin{array}{llll}
\mathrm{H} & -3.739202 & 3.707068 & -1.948977
\end{array} \\
& \begin{array}{llll}
\mathrm{H} & -1.419715 & 2.485126 & -0.595792
\end{array} \\
& \begin{array}{llll}
\mathrm{H} & -3.049299 & 2.140252 & -0.018553
\end{array} \\
& \begin{array}{llll}
\mathrm{H} & -1.910454 & 0.809734 & -0.331265
\end{array} \\
& \begin{array}{llll}
\mathrm{H} & -4.719098 & -2.899896 & -1.154697
\end{array} \\
& \begin{array}{llll}
\mathrm{H} & -3.330881 & -7.644205 & 0.494053
\end{array} \\
& \begin{array}{llll}
\mathrm{H} & -1.211114 & -8.836338 & 0.976712
\end{array} \\
& \begin{array}{llll}
\mathrm{H} & 0.963929 & -7.684575 & 0.775474
\end{array} \\
& \begin{array}{llll}
\mathrm{H} & 1.058481 & -5.292664 & 0.090696
\end{array}
\end{aligned}
$$

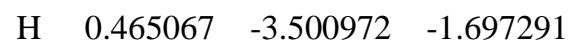

$$
\begin{aligned}
& \begin{array}{llll}
\mathrm{H} & -0.953497 & -4.034835 & -2.619613
\end{array} \\
& \begin{array}{llll}
\mathrm{H} & -0.791023 & -2.337364 & -2.130511
\end{array} \\
& \begin{array}{llll}
\mathrm{H} & 0.189964 & -2.916482 & 0.831623
\end{array} \\
& \begin{array}{llll}
\mathrm{H} & -1.026238 & -1.713684 & 0.383493
\end{array} \\
& \begin{array}{llll}
\mathrm{H} & -1.418129 & -2.989693 & 1.559359
\end{array} \\
& \mathrm{H} \quad-5.320161 \quad-4.390570 \quad 0.070456 \\
& \begin{array}{llll}
\mathrm{H} & -4.801052 & -5.903915 & 0.752464
\end{array} \\
& \begin{array}{llll}
\text { C } & -6.764358 & -6.514350 & -0.863629
\end{array} \\
& \begin{array}{llll}
\text { C } & -7.385352 & -7.609739 & -1.728693
\end{array} \\
& \begin{array}{llll}
\mathrm{H} & -8.361862 & -7.907272 & -1.334135
\end{array} \\
& \begin{array}{llll}
\mathrm{H} & -7.536727 & -7.284385 & -2.762583
\end{array} \\
& \begin{array}{llll}
\mathrm{H} & -6.746209 & -8.499855 & -1.748887
\end{array} \\
& \begin{array}{llll}
\mathrm{H} & -6.701257 & -6.880079 & 0.170113
\end{array} \\
& \begin{array}{lllll}
\mathrm{H} & -7.428745 & -5.639094 & -0.833862
\end{array} \\
& \begin{array}{llll}
\text { C } & -5.446506 & -5.240977 & -2.626007
\end{array} \\
& \begin{array}{llll}
\text { C } & -4.153117 & -5.167201 & -3.442945
\end{array} \\
& \begin{array}{llll}
\text { C } & -4.219867 & -4.113979 & -4.547948
\end{array} \\
& \begin{array}{llll}
\text { C } & -2.932854 & -4.027205 & -5.364335
\end{array} \\
& \mathrm{H} \quad-3.012747 \quad-3.275459 \quad-6.155857 \\
& \begin{array}{llll}
\mathrm{H} & -2.084641 & -3.749790 & -4.727088
\end{array} \\
& \begin{array}{llll}
\mathrm{H} & -2.695560 & -4.988080 & -5.835404
\end{array} \\
& \begin{array}{llll}
\mathrm{H} & -6.223864 & -5.667104 & -3.271177
\end{array} \\
& \begin{array}{llll}
\mathrm{H} & -5.800283 & -4.227916 & -2.388188
\end{array} \\
& \mathrm{H} \quad-3.951290 \quad-6.154762-3.880800
\end{aligned}
$$

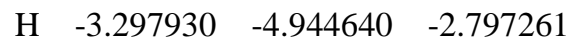

$$
\begin{aligned}
& \text { H } \quad-5.067383 \quad-4.331646 \quad-5.211233 \\
& \begin{array}{llll}
\mathrm{H} & -4.429357 & -3.136023 & -4.094204
\end{array} \\
& \begin{array}{llll}
\mathrm{H} & -4.756818 & -6.954673 & -1.512667
\end{array} \\
& \text { 2xMB:PCBM } \mathrm{t}\left(\mathrm{PC}_{61} \mathrm{BM}\right. \text { on top) } \\
& \begin{array}{llll}
\text { C } & -5.335148 & -3.679957 & 0.818962
\end{array}
\end{aligned}
$$




\begin{tabular}{|c|c|c|c|}
\hline $\mathrm{C}$ & -4.851484 & -2.470829 & \\
\hline $\mathrm{C}$ & -4.941604 & -2.123181 & 2.646784 \\
\hline $\mathrm{C}$ & -5.545740 & -2.984831 & 3.544054 \\
\hline $\mathrm{C}$ & -6.042293 & -4.208426 & 3.081685 \\
\hline C & -5.928014 & -4.548944 & 1.735890 \\
\hline C & -4.251320 & -0.790477 & 2.866500 \\
\hline $\mathrm{C}$ & -3.807187 & -0.438788 & 1.439194 \\
\hline $\mathrm{N}$ & -4.179162 & -1.465570 & 0.603483 \\
\hline $\mathrm{C}$ & -3.135003 & 0.667008 & 0.997663 \\
\hline $\mathrm{C}$ & -2.656861 & 1.760922 & 1.770584 \\
\hline $\mathrm{C}$ & -2.038282 & 2.849775 & 1.228349 \\
\hline$S$ & -1.875728 & 3.042841 & -0.520037 \\
\hline $\mathrm{C}$ & -0.960770 & 4.507206 & -0.250622 \\
\hline $\mathrm{N}$ & -0.797643 & 4.825795 & 1.064894 \\
\hline $\mathrm{C}$ & -1.368730 & 3.972349 & 1.870158 \\
\hline $\mathrm{C}$ & -0.398892 & 5.233940 & -1.273759 \\
\hline $\mathrm{C}$ & 0.427102 & 6.362542 & -1.003578 \\
\hline $\mathrm{N}$ & 1.132924 & 7.262879 & -0.808842 \\
\hline $\mathrm{C}$ & -1.257978 & 4.198513 & 3.371120 \\
\hline $\mathrm{C}$ & -2.663858 & 4.329346 & 3.987499 \\
\hline $\mathrm{C}$ & -5.224030 & 0.237464 & 3.464720 \\
\hline $\mathrm{C}$ & -3.023464 & -0.987941 & 3.778699 \\
\hline $\mathrm{C}$ & -3.837826 & -1.593201 & -0.798532 \\
\hline $\mathrm{C}$ & -2.647156 & -2.518582 & -1.131660 \\
\hline $\mathrm{C}$ & -0.471486 & 3.042128 & 4.019847 \\
\hline $\mathrm{C}$ & -0.492305 & 5.503509 & 3.635120 \\
\hline $\mathrm{C}$ & -0.552671 & 4.789952 & -2.616452 \\
\hline $\mathrm{N}$ & -0.696124 & 4.361597 & -3.687507 \\
\hline $\mathrm{H}$ & -2.764804 & 1.731727 & 2.846792 \\
\hline $\mathrm{H}$ & -0.401368 & 3.213842 & 5.099412 \\
\hline $\mathrm{H}$ & -0.932420 & 2.062911 & 3.862903 \\
\hline $\mathrm{H}$ & 0.546120 & 2.998322 & 3.619422 \\
\hline $\mathrm{H}$ & -0.417795 & 5.661589 & 4.716823 \\
\hline $\mathrm{H}$ & 0.516320 & 5.464690 & 3.215431 \\
\hline $\mathrm{H}$ & -1.003636 & 6.359443 & 3.187664 \\
\hline $\mathrm{H}$ & -2.571721 & 4.565180 & 5.053031 \\
\hline $\mathrm{H}$ & -3.217798 & 5.143215 & 3.509105 \\
\hline $\mathrm{H}$ & -3.266156 & 3.421524 & 3.902940 \\
\hline $\mathrm{H}$ & -2.903698 & 0.711723 & -0.061117 \\
\hline $\mathrm{H}$ & -5.239801 & -3.956780 & -0.225154 \\
\hline $\mathrm{H}$ & -6.310919 & -5.502931 & 1.385403 \\
\hline $\mathrm{H}$ & -6.508184 & -4.899003 & 3.777256 \\
\hline $\mathrm{H}$ & -5.620428 & -2.724780 & 4.596560 \\
\hline $\mathrm{H}$ & -3.346953 & -1.371836 & 4.751571 \\
\hline $\mathrm{H}$ & -2.322268 & -1.704686 & 3.342682 \\
\hline $\mathrm{H}$ & -2.490616 & -0.048273 & 3.947598 \\
\hline $\mathrm{H}$ & -5.534924 & -0.091511 & 4.461869 \\
\hline $\mathrm{H}$ & -4.775022 & 1.228570 & 3.563043 \\
\hline
\end{tabular}

\begin{tabular}{|c|c|c|c|}
\hline & & & \\
\hline & -3.644530 & & \\
\hline & & & \\
\hline & & & \\
\hline & & & \\
\hline & & & \\
\hline & & & \\
\hline & & & \\
\hline & & & \\
\hline & & & \\
\hline & & & \\
\hline & & & \\
\hline & & & \\
\hline & -0.6 & & \\
\hline & & & \\
\hline & & & \\
\hline & -0.7 & -5.4 & \\
\hline & & & \\
\hline & & & \\
\hline & & & \\
\hline & -2.5 & & \\
\hline & & & \\
\hline & -0.3 & & \\
\hline & -3.0 & & \\
\hline & 12.0 & -3.7 & \\
\hline & $11.5 \mathrm{~s}$ & & \\
\hline & & & \\
\hline & & & \\
\hline & & & \\
\hline & & & \\
\hline & & & \\
\hline & & -2.3 & \\
\hline & & & \\
\hline C & & -1.0 & \\
\hline & & & \\
\hline & & & \\
\hline & & & \\
\hline & & & \\
\hline & & $2.89^{\circ}$ & 2.2 \\
\hline & & & \\
\hline $\mathrm{C}$ & & 2.65 & \\
\hline & & & \\
\hline & & 1.819 & -1.75 \\
\hline & & 0.525820 & \\
\hline & & & \\
\hline & & 1.424125 & \\
\hline & & & \\
\hline & 2.931202 & 1.597705 & \\
\hline
\end{tabular}




\begin{tabular}{|c|c|c|c|}
\hline $\mathrm{C}$ & 4.143768 & 0.918749 & 3.190080 \\
\hline C & 4.282522 & -0.450180 & 2.989390 \\
\hline C & 3.202031 & -1.196844 & 2.378008 \\
\hline $\mathrm{C}$ & 2.039693 & -0.548585 & 1.991807 \\
\hline C & 1.410575 & -0.882340 & 0.729997 \\
\hline C & 1.960986 & -1.860635 & -0.081387 \\
\hline C & 3.167853 & -2.547619 & 0.328160 \\
\hline $\mathrm{C}$ & 3.783821 & -2.217399 & 1.530138 \\
\hline C & 3.971489 & -2.763923 & -0.845195 \\
\hline $\mathrm{C}$ & 5.350784 & -2.636716 & -0.756078 \\
\hline $\mathrm{C}$ & 6.086830 & -1.974565 & -1.812042 \\
\hline $\mathrm{C}$ & 7.192012 & -1.267085 & -1.221400 \\
\hline $\mathrm{C}$ & 7.535876 & -0.025960 & -1.710613 \\
\hline $\mathrm{C}$ & 7.848391 & 1.086817 & -0.793345 \\
\hline $\mathrm{C}$ & 7.802550 & 0.907398 & 0.571534 \\
\hline $\mathrm{C}$ & 7.180891 & 1.910868 & 1.393826 \\
\hline $\mathrm{C}$ & 6.692675 & 3.087937 & 0.843772 \\
\hline $\mathrm{C}$ & 5.443890 & 3.650390 & 1.321030 \\
\hline $\mathrm{C}$ & 4.741322 & 4.198057 & 0.178544 \\
\hline $\mathrm{C}$ & 3.362985 & 4.081873 & 0.096149 \\
\hline $\mathrm{C}$ & 2.743459 & 3.735611 & -1.165799 \\
\hline $\mathrm{C}$ & 3.521538 & 3.526897 & -2.292511 \\
\hline $\mathrm{C}$ & 4.961450 & 3.643974 & -2.204657 \\
\hline $\mathrm{C}$ & 5.559089 & 3.972160 & -0.998028 \\
\hline $\mathrm{C}$ & 6.765700 & 3.287680 & -0.579800 \\
\hline $\mathrm{C}$ & 7.320682 & 2.296173 & -1.379907 \\
\hline $\mathrm{C}$ & 6.693833 & 1.957333 & -2.640722 \\
\hline $\mathrm{C}$ & 5.544155 & 2.616207 & -3.045457 \\
\hline $\mathrm{C}$ & 4.461019 & 1.873359 & -3.662331 \\
\hline $\mathrm{C}$ & 3.211660 & 2.433266 & -3.194199 \\
\hline C & 2.133719 & 1.601035 & -2.933028 \\
\hline $\mathrm{C}$ & 2.257318 & 0.171794 & -3.131767 \\
\hline $\mathrm{C}$ & 1.520034 & -0.493577 & -2.073618 \\
\hline $\mathrm{C}$ & 2.015686 & -1.664239 & -1.515566 \\
\hline $\mathrm{C}$ & 3.265048 & -2.222272 & -1.990789 \\
\hline $\mathrm{C}$ & 3.968109 & -1.585502 & -2.999264 \\
\hline C & 3.454655 & -0.363757 & -3.584477 \\
\hline C & 4.581935 & 0.506499 & -3.856694 \\
\hline $\mathrm{C}$ & 5.789317 & -0.179490 & -3.440290 \\
\hline $\mathrm{C}$ & 5.411184 & -1.463230 & -2.911617 \\
\hline $\mathrm{C}$ & 6.822702 & 0.527932 & -2.837967 \\
\hline $\mathrm{C}$ & 8.530958 & -2.582164 & 2.254905 \\
\hline $\mathrm{C}$ & 8.257311 & -3.939312 & 2.085362 \\
\hline $\mathrm{C}$ & 8.354633 & -4.817323 & 3.160549 \\
\hline $\mathrm{C}$ & 8.724695 & -4.344736 & 4.416067 \\
\hline $\mathrm{C}$ & 9.000228 & -2.991106 & 4.592311 \\
\hline $\mathrm{C}$ & 8.903938 & -2.115986 & 3.516188 \\
\hline $\mathrm{C}$ & 13.703524 & -4.620758 & -3.240315 \\
\hline
\end{tabular}

\begin{tabular}{|c|c|c|c|}
\hline $\mathrm{H}$ & 11.511570 & -1.676081 & \\
\hline $\mathrm{H}$ & 12.398704 & -2.219402 & -0.412656 \\
\hline $\mathrm{H}$ & 9.502230 & -3.001055 & -1.077283 \\
\hline $\mathrm{H}$ & 10.385258 & -3.556957 & 0.332925 \\
\hline $\mathrm{H}$ & 9.803693 & -0.614542 & -0.301358 \\
\hline $\mathrm{H}$ & 10.586166 & -1.197094 & 1.158951 \\
\hline $\mathrm{H}$ & 7.963010 & -4.308969 & 1.107082 \\
\hline $\mathrm{H}$ & 8.138511 & -5.871417 & 3.015625 \\
\hline $\mathrm{H}$ & 8.796194 & -5.028835 & 5.256297 \\
\hline $\mathrm{H}$ & 9.286367 & -2.615112 & 5.569955 \\
\hline $\mathrm{H}$ & 9.110449 & -1.057689 & 3.654520 \\
\hline $\mathrm{H}$ & 14.639124 & -4.276537 & -3.680249 \\
\hline $\mathrm{H}$ & 12.995986 & -4.908298 & -4.022056 \\
\hline $\mathrm{H}$ & 13.880193 & -5.480614 & -2.589241 \\
\hline $\mathrm{O}$ & 13.201447 & -3.516310 & -2.489403 \\
\hline $\mathrm{O}$ & 11.436990 & -4.781325 & -1.906282 \\
\hline $\mathrm{C}$ & -9.594841 & -5.168183 & -0.478323 \\
\hline $\mathrm{C}$ & -8.901754 & -4.095022 & -1.020235 \\
\hline C & -8.557026 & -4.044832 & -2.367987 \\
\hline $\mathrm{C}$ & -8.893809 & -5.087034 & -3.210966 \\
\hline $\mathrm{C}$ & -9.584859 & -6.183444 & -2.686633 \\
\hline $\mathrm{C}$ & -9.929158 & -6.216596 & -1.337999 \\
\hline $\mathrm{C}$ & -7.830514 & -2.745358 & -2.649298 \\
\hline $\mathrm{C}$ & -7.802100 & -2.107485 & -1.252711 \\
\hline $\mathrm{N}$ & -8.441530 & -2.938095 & -0.375993 \\
\hline C & -7.297090 & -0.891983 & -0.878426 \\
\hline $\mathrm{C}$ & -6.638945 & 0.055878 & -1.700468 \\
\hline $\mathrm{C}$ & -6.252826 & 1.289956 & -1.262480 \\
\hline$S$ & -6.614840 & 1.831774 & 0.380691 \\
\hline $\mathrm{C}$ & -5.640431 & 3.258110 & 0.091753 \\
\hline $\mathrm{N}$ & -5.185564 & 3.366939 & -1.189339 \\
\hline $\mathrm{C}$ & -5.488511 & 2.331429 & -1.927283 \\
\hline $\mathrm{C}$ & -5.341545 & 4.162414 & 1.083266 \\
\hline $\mathrm{C}$ & -4.512557 & 5.293070 & 0.833879 \\
\hline $\mathrm{N}$ & -3.823329 & 6.214136 & 0.678289 \\
\hline $\mathrm{C}$ & -5.005313 & 2.291802 & -3.372379 \\
\hline $\mathrm{C}$ & -3.983288 & 1.148362 & -3.541385 \\
\hline $\mathrm{C}$ & -6.422507 & -3.026971 & -3.202678 \\
\hline $\mathrm{C}$ & -8.646399 & -1.886161 & -3.633882 \\
\hline $\mathrm{C}$ & -8.614012 & -2.737906 & 1.054575 \\
\hline $\mathrm{C}$ & -9.962418 & -2.154741 & 1.513963 \\
\hline $\mathrm{C}$ & -6.198017 & 2.104744 & -4.331491 \\
\hline $\mathrm{C}$ & -4.309915 & 3.615610 & -3.723044 \\
\hline $\mathrm{C}$ & -5.794105 & 3.924445 & 2.410551 \\
\hline $\mathrm{N}$ & -6.166213 & 3.672233 & 3.482636 \\
\hline $\mathrm{H}$ & -6.406950 & -0.205247 & -2.724489 \\
\hline $\mathrm{H}$ & -5.839724 & 2.160331 & -5.364612 \\
\hline $\mathrm{H}$ & -6.708803 & 1.145491 & -4.209659 \\
\hline
\end{tabular}




\begin{tabular}{|c|c|c|c|}
\hline $\mathrm{H}$ & -6.937807 & 2.898914 & -4.187870 \\
\hline $\mathrm{H}$ & -3.962275 & 3.576897 & -4.760423 \\
\hline $\mathrm{H}$ & -4.992035 & 4.462888 & -3.612547 \\
\hline $\mathrm{H}$ & -3.444933 & 3.800265 & -3.084109 \\
\hline $\mathrm{H}$ & -3.583302 & 1.165182 & -4.560320 \\
\hline $\mathrm{H}$ & -3.139493 & 1.278622 & -2.854652 \\
\hline $\mathrm{H}$ & -4.417752 & 0.158821 & -3.367399 \\
\hline $\mathrm{H}$ & -7.428980 & -0.610544 & 0.159141 \\
\hline $\mathrm{H}$ & -9.879730 & -5.196890 & 0.568065 \\
\hline $\mathrm{H}$ & -10.471845 & -7.069841 & -0.943162 \\
\hline $\mathrm{H}$ & -9.857858 & -7.009748 & -3.334629 \\
\hline $\mathrm{H}$ & -8.628767 & -5.058079 & -4.264606 \\
\hline $\mathrm{H}$ & -8.727132 & -2.404946 & -4.594549 \\
\hline $\mathrm{H}$ & -9.657496 & -1.714240 & -3.254533 \\
\hline $\mathrm{H}$ & -8.177054 & -0.913716 & -3.808381 \\
\hline $\mathrm{H}$ & -6.504357 & -3.578192 & -4.144830 \\
\hline $\mathrm{H}$ & -5.866229 & -2.106914 & -3.404694 \\
\hline $\mathrm{H}$ & -5.840115 & -3.638941 & -2.507308 \\
\hline $\mathrm{H}$ & -7.785328 & -2.127023 & 1.425716 \\
\hline $\mathrm{H}$ & -8.473660 & -3.71 & 4752 \\
\hline $\mathrm{C}$ & -10.031214 & -2.316047 & 3.044476 \\
\hline $\mathrm{C}$ & -11.432646 & -2.147427 & 3.629117 \\
\hline $\mathrm{H}$ & -11.420095 & -2.298213 & 4.712977 \\
\hline $\mathrm{H}$ & -11.841361 & -1.149375 & 3.443142 \\
\hline $\mathrm{H}$ & -12.125857 & -2.877841 & 3.196520 \\
\hline $\mathrm{H}$ & -9.657617 & -3.311356 & 3.320415 \\
\hline $\mathrm{H}$ & -9.343161 & -1.595123 & 3.507800 \\
\hline $\mathrm{C}$ & -10.168619 & -0.688191 & 1.096267 \\
\hline $\mathrm{C}$ & -10.665108 & -0.472199 & -0.336023 \\
\hline $\mathrm{C}$ & -10.535220 & 0.981849 & -0.787502 \\
\hline C & -10.993344 & 1.201510 & -2.227024 \\
\hline $\mathrm{H}$ & -10.907103 & 2.253562 & -2.516413 \\
\hline $\mathrm{H}$ & -10.383416 & 0.613902 & -2.923542 \\
\hline $\mathrm{H}$ & -12.038496 & 0.900110 & -2.362608 \\
\hline $\mathrm{H}$ & -10.888960 & -0.216527 & 1.774849 \\
\hline $\mathrm{H}$ & -9.228973 & -0.140278 & 1.254389 \\
\hline $\mathrm{H}$ & -11.714197 & -0.792287 & -0.403367 \\
\hline $\mathrm{H}$ & -10.111389 & -1.107404 & -1.033886 \\
\hline $\mathrm{H}$ & -11.109422 & 1.628227 & -0.110770 \\
\hline $\mathrm{H}$ & -9.486202 & 1.291666 & -0.689771 \\
\hline $\mathrm{H}$ & -10.763641 & -2.764630 & 1.069152 \\
\hline
\end{tabular}

2xML:PC ${ }_{61} \mathrm{BM} \mathrm{t}\left(\mathrm{PC}_{61} \mathrm{BM}\right.$ on top) cluster
$\begin{array}{llll}\text { C } & 2.467306 & -1.899523 & -1.955914\end{array}$
$\begin{array}{llll}\text { S } & 2.392220 & -0.186952 & -2.307101\end{array}$
$\begin{array}{llll}\text { C } & 2.928140 & 0.140682 & -0.651787\end{array}$
$\begin{array}{llll}\text { C } & 3.209007 & -1.116089 & -0.007727\end{array}$

\begin{tabular}{|c|c|c|c|}
\hline & & & \\
\hline & & & \\
\hline & & & \\
\hline & & & \\
\hline & & & \\
\hline & & & \\
\hline & & & \\
\hline & & & \\
\hline & & & \\
\hline & & & \\
\hline & & & \\
\hline & & & \\
\hline & & & \\
\hline & & & \\
\hline & & & \\
\hline & & & \\
\hline & & & \\
\hline & & & \\
\hline & & & \\
\hline & & & \\
\hline & & & \\
\hline & & & \\
\hline & & & \\
\hline & & & \\
\hline & & & \\
\hline & & & \\
\hline & & & \\
\hline & & & \\
\hline & & & \\
\hline & & & \\
\hline & & & \\
\hline & & & \\
\hline & & & \\
\hline & 1.35 & & \\
\hline & & & \\
\hline & & & \\
\hline & & & \\
\hline & 2.25 & & \\
\hline & & & \\
\hline & & 53 & \\
\hline & & & \\
\hline & & & \\
\hline & & & \\
\hline & & & \\
\hline & & 4.749289 & \\
\hline & & & \\
\hline & & 3.303633 & \\
\hline & -0.347766 & 2.264396 & -4.6797 \\
\hline
\end{tabular}




\begin{tabular}{|c|c|c|c|}
\hline $\mathrm{C}$ & -0.156946 & 1.501533 & -5.988659 \\
\hline C & -1.136247 & 0.334950 & -6.108986 \\
\hline $\mathrm{C}$ & -0.974669 & -0.482122 & -7.387153 \\
\hline C & -1.877402 & -1.715205 & -7.408520 \\
\hline $\mathrm{C}$ & -1.724648 & -2.542831 & -8.682853 \\
\hline $\mathrm{H}$ & -2.377480 & -3.422200 & -8.671417 \\
\hline $\mathrm{H}$ & -1.975112 & -1.950464 & -9.571148 \\
\hline $\mathrm{H}$ & -0.692354 & -2.893154 & -8.796116 \\
\hline $\mathrm{H}$ & 2.694102 & 4.251151 & -2.962526 \\
\hline $\mathrm{H}$ & 1.810646 & 5.760776 & -2.993590 \\
\hline $\mathrm{H}$ & 0.317728 & 3.209572 & -2.279318 \\
\hline $\mathrm{H}$ & -0.327212 & 4.648187 & -3.039678 \\
\hline $\mathrm{H}$ & 0.771261 & 4.056637 & -5.186421 \\
\hline $\mathrm{H}$ & 1.708524 & 2.795014 & -4.397639 \\
\hline $\mathrm{H}$ & -0.343173 & 1.536321 & -3.860158 \\
\hline $\mathrm{H}$ & -1.338976 & 2.738230 & -4.663958 \\
\hline $\mathrm{H}$ & -0.266822 & 2.182510 & -6.844646 \\
\hline $\mathrm{H}$ & 0.867250 & 1.104922 & -6.031344 \\
\hline $\mathrm{H}$ & -2.168213 & 0.709455 & -6.033382 \\
\hline $\mathrm{H}$ & -0.985088 & -0.332891 & -5.251475 \\
\hline $\mathrm{H}$ & -1.184636 & 0.147946 & -8.264264 \\
\hline $\mathrm{H}$ & 0.070182 & -0.807876 & -7.469813 \\
\hline $\mathrm{H}$ & -2.925112 & -1.404004 & -7.293076 \\
\hline $\mathrm{H}$ & -1.637143 & -2.336735 & -6.536514 \\
\hline $\mathrm{C}$ & -12.508683 & -2.388904 & 0.615801 \\
\hline $\mathrm{C}$ & -11.422431 & -2.045242 & 1.609709 \\
\hline $\mathrm{C}$ & -10.498584 & -0.944457 & 1.103584 \\
\hline $\mathrm{C}$ & -9.406069 & -0.611911 & 2.118054 \\
\hline $\mathrm{C}$ & -8.428630 & 0.438441 & 1.600109 \\
\hline $\mathrm{C}$ & -6.993045 & 0.356970 & 2.057468 \\
\hline $\mathrm{C}$ & -7.429768 & 0.003449 & 0.559961 \\
\hline $\mathrm{C}$ & -6.831213 & 0.857280 & -0.502228 \\
\hline $\mathrm{C}$ & -6.084779 & 1.982736 & -0.237426 \\
\hline $\mathrm{C}$ & -5.681272 & 2.309638 & 1.142403 \\
\hline $\mathrm{C}$ & -6.044743 & 1.497910 & 2.193389 \\
\hline $\mathrm{C}$ & -5.073265 & 1.167853 & 3.200875 \\
\hline $\mathrm{C}$ & -3.795560 & 1.709865 & 3.172392 \\
\hline $\mathrm{C}$ & -2.650657 & 0.887709 & 3.516065 \\
\hline $\mathrm{C}$ & -1.562748 & 1.252670 & 2.631858 \\
\hline $\mathrm{C}$ & -0.705337 & 0.272921 & 2.150452 \\
\hline $\mathrm{C}$ & -0.284446 & 0.296840 & 0.765741 \\
\hline $\mathrm{C}$ & -0.214293 & -1.071979 & 0.293993 \\
\hline C & -0.599687 & -1.380431 & -0.999954 \\
\hline $\mathrm{C}$ & -1.071924 & -0.334858 & -1.882627 \\
\hline $\mathrm{C}$ & -1.143658 & 0.973084 & -1.431174 \\
\hline C & -0.738424 & 1.299373 & -0.078400 \\
\hline $\mathrm{C}$ & -1.636868 & 2.317928 & 0.421025 \\
\hline $\mathrm{C}$ & -2.041487 & 2.296848 & 1.747103 \\
\hline
\end{tabular}

\begin{tabular}{|c|c|c|c|}
\hline $\mathrm{C}$ & -3.421917 & 2.578094 & 2.087950 \\
\hline $\mathrm{C}$ & -4.345833 & 2.855742 & 1.087009 \\
\hline $\mathrm{C}$ & -3.918490 & 2.879206 & -0.297257 \\
\hline C & -2.596886 & 2.621238 & -0.623576 \\
\hline C & -2.284304 & 1.793818 & -1.771230 \\
\hline C & -3.303070 & 1.274590 & -2.553860 \\
\hline C & -4.684241 & 1.555045 & -2.218135 \\
\hline C & -4.988793 & 2.335176 & -1.108173 \\
\hline $\mathrm{C}$ & -5.453086 & 0.367467 & -2.480633 \\
\hline $\mathrm{C}$ & -6.486413 & 0.022710 & -1.620520 \\
\hline C & -6.679027 & -1.359580 & -1.236898 \\
\hline $\mathrm{C}$ & -7.149378 & -1.393127 & 0.122724 \\
\hline C & -6.687359 & -2.374332 & 0.972092 \\
\hline $\mathrm{C}$ & -6.280291 & -2.046007 & 2.351796 \\
\hline $\mathrm{C}$ & -6.357046 & -0.752782 & 2.819932 \\
\hline $\mathrm{C}$ & -5.264208 & -0.214616 & 3.585298 \\
\hline C & -4.169818 & -0.998450 & 3.922933 \\
\hline $\mathrm{C}$ & -2.833651 & -0.435109 & 3.882188 \\
\hline $\mathrm{C}$ & -1.938521 & -1.458770 & 3.383171 \\
\hline $\mathrm{C}$ & -0.898164 & -1.112654 & 2.534496 \\
\hline $\mathrm{C}$ & -0.594281 & -1.943072 & 1.386919 \\
\hline C & -1.343640 & -3.082510 & 1.135745 \\
\hline $\mathrm{C}$ & -2.432691 & -3.439779 & 2.019289 \\
\hline C & -2.724052 & -2.648573 & 3.119342 \\
\hline $\mathrm{C}$ & -4.103035 & -2.358571 & 3.457589 \\
\hline C & -5.133978 & -2.862580 & 2.674132 \\
\hline $\mathrm{C}$ & -4.825718 & -3.691440 & 1.526904 \\
\hline $\mathrm{C}$ & -3.508202 & -3.977779 & 1.207860 \\
\hline $\mathrm{C}$ & -3.076268 & -3.963349 & -0.176609 \\
\hline $\mathrm{C}$ & -1.740953 & -3.406270 & -0.221562 \\
\hline $\mathrm{C}$ & -1.372554 & -2.575199 & -1.267837 \\
\hline $\mathrm{C}$ & -2.320513 & -2.266749 & -2.319615 \\
\hline $\mathrm{C}$ & -2.131840 & -0.880459 & -2.700709 \\
\hline $\mathrm{C}$ & -3.224346 & -0.092302 & -3.029914 \\
\hline $\mathrm{C}$ & -4.557514 & -0.655828 & -2.985986 \\
\hline $\mathrm{C}$ & -4.739427 & -1.978066 & -2.617054 \\
\hline $\mathrm{C}$ & -3.597954 & -2.803369 & -2.277828 \\
\hline $\mathrm{C}$ & -3.985010 & -3.669947 & -1.180857 \\
\hline $\mathrm{C}$ & -5.365103 & -3.379928 & -0.846375 \\
\hline C & -5.826343 & -2.339061 & -1.726710 \\
\hline C & -5.778757 & -3.383790 & 0.480306 \\
\hline $\mathrm{C}$ & -9.025763 & 1.813187 & 1.465436 \\
\hline $\mathrm{C}$ & -9.609623 & 2.229903 & 0.269289 \\
\hline $\mathrm{C}$ & -10.215043 & 3.479544 & 0.179035 \\
\hline $\mathrm{C}$ & -10.239778 & 4.324318 & 1.284634 \\
\hline $\mathrm{C}$ & -9.659719 & 3.914120 & 2.482368 \\
\hline $\mathrm{C}$ & -9.056750 & 2.664404 & 2.570735 \\
\hline $\mathrm{C}$ & -14.361373 & -3.764023 & 0.219830 \\
\hline
\end{tabular}




\begin{tabular}{|c|c|c|c|}
\hline $\mathrm{H}$ & -10.862790 & -2.963346 & 1.828375 \\
\hline & -11.904959 & -1.758046 & 2.552348 \\
\hline H & -10.039745 & -1.264957 & 0.160860 \\
\hline H & -11.088463 & -0.052001 & 0.871977 \\
\hline $\mathrm{H}$ & -8.868597 & -1.527144 & 2.386403 \\
\hline $\mathrm{H}$ & -9.850217 & -0.228568 & 3.045305 \\
\hline $\mathrm{H}$ & -9.586625 & 1.573598 & -0.596171 \\
\hline $\mathrm{H}$ & -10.665528 & 3.792675 & -0.757822 \\
\hline $\mathrm{H}$ & -10.708058 & 5.301345 & 1.213007 \\
\hline $\mathrm{H}$ & -9.673021 & 4.569596 & 3.347889 \\
\hline $\mathrm{H}$ & -8.596646 & 2.346267 & 3.502925 \\
\hline $\mathrm{H}$ & -14.896433 & -4.550699 & 0.751002 \\
\hline $\mathrm{H}$ & -13.961631 & -4.142990 & -0.724039 \\
\hline $\mathrm{H}$ & -15.026629 & -2.922685 & 0.010158 \\
\hline $\mathrm{O}$ & -13.302848 & -3.363260 & 1.089055 \\
\hline $\mathrm{O}$ & -12.660545 & -1.871008 & -0.464917 \\
\hline $\mathrm{C}$ & 7.365309 & 3.376590 & 1.088487 \\
\hline$S$ & 7.933159 & 1.717640 & 1.076159 \\
\hline C & 7.384350 & 1.575317 & -0.600352 \\
\hline C & 6.833713 & 2.843690 & -1.010250 \\
\hline $\mathrm{N}$ & 6.832820 & 3.785391 & -0.096740 \\
\hline $\mathrm{C}$ & 7.459003 & 0.400532 & -1.300942 \\
\hline C & 7.967729 & -0.817237 & -0.803673 \\
\hline $\mathrm{C}$ & 6.318800 & 3.191535 & -2.405556 \\
\hline $\mathrm{C}$ & 5.347055 & 2.125471 & -2.961906 \\
\hline $\mathrm{C}$ & 7.436335 & 4.187595 & 2.201953 \\
\hline $\mathrm{C}$ & 7.970507 & 3.685056 & 3.420678 \\
\hline $\mathrm{N}$ & 8.405324 & 3.228181 & 4.396650 \\
\hline $\mathrm{C}$ & 5.583486 & 4.540078 & -2.346965 \\
\hline $\mathrm{C}$ & 7.539139 & 3.326253 & -3.339648 \\
\hline $\mathrm{C}$ & 6.955146 & 5.527256 & 2.157728 \\
\hline $\mathrm{N}$ & 6.547139 & 6.614370 & 2.142411 \\
\hline $\mathrm{H}$ & 7.067566 & 0.420089 & -2.307126 \\
\hline $\mathrm{H}$ & 4.656768 & 2.591867 & -3.672790 \\
\hline $\mathrm{H}$ & 5.858472 & 1.330306 & -3.513028 \\
\hline $\mathrm{H}$ & 4.753220 & 1.664322 & -2.169887 \\
\hline $\mathrm{H}$ & 5.251623 & 4.812031 & -3.355450 \\
\hline $\mathrm{H}$ & 4.706884 & 4.476122 & -1.694717 \\
\hline $\mathrm{H}$ & 6.223469 & 5.334697 & -1.959517 \\
\hline $\mathrm{H}$ & 7.201733 & 3.585109 & -4.349346 \\
\hline $\mathrm{H}$ & 8.213211 & 4.114660 & -2.990000 \\
\hline $\mathrm{H}$ & 8.107642 & 2.392015 & -3.397740 \\
\hline $\mathrm{C}$ & 7.876623 & -2.035831 & -1.441988 \\
\hline $\mathrm{C}$ & 7.204705 & -2.363776 & -2.781250 \\
\hline $\mathrm{N}$ & 8.355484 & -3.185595 & -0.898887 \\
\hline $\mathrm{C}$ & 8.051304 & -4.300437 & -1.704461 \\
\hline C & 7.394341 & -3.864971 & -2.849793 \\
\hline $\mathrm{H}$ & 8.410480 & -0.797210 & 0.187138 \\
\hline
\end{tabular}

\begin{tabular}{|c|c|c|c|}
\hline $\mathrm{C}$ & 7.895116 & -1.671782 & -3.969571 \\
\hline $\mathrm{C}$ & 5.698154 & -2.048268 & -2.726560 \\
\hline $\mathrm{C}$ & 6.988151 & -4.776979 & -3.806086 \\
\hline $\mathrm{C}$ & 7.249911 & -6.133037 & -3.596383 \\
\hline $\mathrm{C}$ & 7.902791 & -6.553828 & -2.440861 \\
\hline $\mathrm{C}$ & 8.315334 & -5.640772 & -1.468661 \\
\hline $\mathrm{H}$ & 8.809484 & -5.982928 & -0.565572 \\
\hline $\mathrm{H}$ & 8.089140 & -7.611476 & -2.284096 \\
\hline $\mathrm{H}$ & 6.929494 & -6.863443 & -4.331585 \\
\hline $\mathrm{H}$ & 6.461693 & -4.450642 & -4.698661 \\
\hline $\mathrm{H}$ & 5.216781 & -2.384787 & -3.650179 \\
\hline $\mathrm{H}$ & 5.224002 & -2.572912 & -1.892312 \\
\hline $\mathrm{H}$ & 5.502120 & -0.978286 & -2.612845 \\
\hline $\mathrm{H}$ & 7.460707 & -2.039250 & -4.904460 \\
\hline $\mathrm{H}$ & 7.766406 & -0.585935 & -3.946852 \\
\hline $\mathrm{H}$ & 8.966852 & -1.890079 & -3.981277 \\
\hline $\mathrm{C}$ & 8.931903 & -3.294997 & 0.431179 \\
\hline $\mathrm{C}$ & 7.854813 & -3.355388 & 1.514632 \\
\hline $\mathrm{C}$ & 8.429468 & -3.371972 & 2.928831 \\
\hline $\mathrm{C}$ & 7.334775 & -3.329078 & 3.995029 \\
\hline $\mathrm{C}$ & 7.874500 & -3.350305 & 5.423895 \\
\hline $\mathrm{C}$ & 6.770733 & -3.273358 & 6.478420 \\
\hline $\mathrm{C}$ & 7.301862 & -3.293862 & 7.911260 \\
\hline $\mathrm{C}$ & 6.197879 & -3.210008 & 8.965704 \\
\hline $\mathrm{C}$ & 6.738199 & -3.232497 & 10.394349 \\
\hline $\mathrm{H}$ & 5.929040 & -3.170743 & 11.129334 \\
\hline $\mathrm{H}$ & 7.297804 & -4.154950 & 10.588190 \\
\hline $\mathrm{H}$ & 7.416483 & -2.389768 & 10.570603 \\
\hline $\mathrm{H}$ & 9.603753 & -2.445331 & 0.589459 \\
\hline $\mathrm{H}$ & 9.560523 & -4.189323 & 0.451265 \\
\hline $\mathrm{H}$ & 7.194318 & -2.487899 & 1.402496 \\
\hline $\mathrm{H}$ & 7.231159 & -4.242665 & 1.346896 \\
\hline $\mathrm{H}$ & 9.048262 & -4.268976 & 3.070848 \\
\hline $\mathrm{H}$ & 9.097419 & -2.509596 & 3.063595 \\
\hline $\mathrm{H}$ & 6.732853 & -2.420430 & 3.850998 \\
\hline $\mathrm{H}$ & 6.650030 & -4.176342 & 3.848427 \\
\hline $\mathrm{H}$ & 8.463651 & -4.265435 & 5.577863 \\
\hline $\mathrm{H}$ & 8.568485 & -2.509681 & 5.562607 \\
\hline $\mathrm{H}$ & 6.072910 & -4.111014 & 6.336618 \\
\hline $\mathrm{H}$ & 6.184998 & -2.356279 & 6.322095 \\
\hline $\mathrm{H}$ & 7.886059 & -4.211723 & 8.069884 \\
\hline $\mathrm{H}$ & 8.001293 & -2.457578 & 8.051396 \\
\hline $\mathrm{H}$ & 5.498172 & -4.044604 & 8.823675 \\
\hline $\mathrm{H}$ & 5.616800 & -2.291774 & 8.807035 \\
\hline \multicolumn{4}{|c|}{$\mathrm{ML}: \mathrm{PC}_{61} \mathrm{BM} \mathrm{s}\left(\mathrm{PC}_{61} \mathrm{BM}\right.$ on side) cluster } \\
\hline $\mathrm{C}$ & 890122 & 1.404633 & -1.907708 \\
\hline
\end{tabular}




\begin{tabular}{|c|c|c|c|}
\hline S & -6.886963 & -0.324576 & -1.628571 \\
\hline C & -5.147945 & -0.236561 & -1.299040 \\
\hline C & -4.720729 & 1.125220 & -1.480162 \\
\hline N & -5.663432 & 1.981888 & -1.804851 \\
\hline $\mathrm{C}$ & -4.437021 & -1.341130 & -0.893141 \\
\hline C & -5.021199 & -2.594074 & -0.638624 \\
\hline $\mathrm{C}$ & -3.296545 & 1.640849 & -1.312208 \\
\hline C & -2.358299 & 0.928313 & -2.304574 \\
\hline $\mathrm{C}$ & -8.043095 & 2.118871 & -2.170054 \\
\hline C & -9.294088 & 1.448140 & -2.239681 \\
\hline $\mathrm{N}$ & -10.294302 & 0.857181 & -2.275582 \\
\hline C & -3.257246 & 3.147252 & -1.612284 \\
\hline $\mathrm{C}$ & -2.827359 & 1.430535 & 0.140470 \\
\hline $\mathrm{C}$ & -8.008020 & 3.536601 & -2.294545 \\
\hline $\mathrm{N}$ & -8.003903 & 4.696342 & -2.357228 \\
\hline $\mathrm{H}$ & -3.371812 & -1.219317 & -0.745442 \\
\hline $\mathrm{H}$ & -1.370008 & 1.394710 & -2.263027 \\
\hline $\mathrm{H}$ & -2.227851 & -0.135117 & -2.086209 \\
\hline $\mathrm{H}$ & -2.731992 & 1.020610 & -3.329545 \\
\hline $\mathrm{H}$ & -2.231449 & 3.511078 & -1.485307 \\
\hline $\mathrm{H}$ & -3.581717 & 3.361495 & -2.633633 \\
\hline $\mathrm{H}$ & -3.910409 & 3.707034 & -0.937415 \\
\hline $\mathrm{H}$ & -1.837893 & 1.880734 & 0.273300 \\
\hline $\mathrm{H}$ & -3.520063 & 1.910583 & 0.838794 \\
\hline $\mathrm{H}$ & -2.744081 & 0.378035 & 0.425501 \\
\hline $\mathrm{C}$ & -4.362192 & -3.752337 & -0.279426 \\
\hline $\mathrm{C}$ & -2.865559 & -3.986354 & -0.086846 \\
\hline $\mathrm{N}$ & -5.029812 & -4.905131 & -0.024889 \\
\hline C & -4.140477 & -5.952295 & 0.293209 \\
\hline $\mathrm{C}$ & -2.841370 & -5.456263 & 0.273818 \\
\hline $\mathrm{H}$ & -6.099740 & -2.647255 & -0.731243 \\
\hline $\mathrm{C}$ & -2.333177 & -3.156463 & 1.094768 \\
\hline $\mathrm{C}$ & -2.075214 & -3.734800 & -1.382444 \\
\hline $\mathrm{C}$ & -1.775865 & -6.281815 & 0.580060 \\
\hline $\mathrm{C}$ & -2.032485 & -7.618973 & 0.890881 \\
\hline C & -3.337576 & -8.105317 & 0.890588 \\
\hline $\mathrm{C}$ & -4.420345 & -7.276625 & 0.590308 \\
\hline $\mathrm{H}$ & -5.431818 & -7.668938 & 0.591888 \\
\hline $\mathrm{H}$ & -3.522494 & -9.147311 & 1.131947 \\
\hline $\mathrm{H}$ & -1.210175 & -8.281283 & 1.139902 \\
\hline $\mathrm{H}$ & -0.760076 & -5.895988 & 0.590128 \\
\hline $\mathrm{H}$ & -1.039015 & -4.056657 & -1.246322 \\
\hline $\mathrm{H}$ & -2.501196 & -4.300102 & -2.216669 \\
\hline $\mathrm{H}$ & -2.063515 & -2.675453 & -1.653023 \\
\hline $\mathrm{H}$ & -1.323947 & -3.488116 & 1.347620 \\
\hline $\mathrm{H}$ & -2.289034 & -2.088103 & 0.858729 \\
\hline $\mathrm{H}$ & -2.955209 & -3.287417 & 1.982989 \\
\hline $\mathrm{C}$ & -6.481180 & -5.003050 & -0.015373 \\
\hline
\end{tabular}

\begin{tabular}{|c|c|c|c|}
\hline $\mathrm{C}$ & -7.117844 & -4.920938 & -1.415385 \\
\hline $\mathrm{C}$ & -8.447892 & -4.163568 & -1.394200 \\
\hline $\mathrm{C}$ & -8.968331 & -3.809125 & -2.785831 \\
\hline $\mathrm{C}$ & -10.258812 & -2.990655 & -2.744490 \\
\hline C & -10.724387 & -2.529211 & -4.124741 \\
\hline $\mathrm{C}$ & -12.037525 & -1.748993 & -4.079822 \\
\hline $\mathrm{C}$ & -12.479952 & -1.236907 & -5.450437 \\
\hline $\mathrm{C}$ & -13.790806 & -0.454548 & -5.393330 \\
\hline $\mathrm{H}$ & -14.086364 & -0.092128 & -6.383740 \\
\hline $\mathrm{H}$ & -14.604976 & -1.079831 & -5.008065 \\
\hline $\mathrm{H}$ & -13.696640 & 0.413245 & -4.730875 \\
\hline $\mathrm{H}$ & -6.854925 & -4.187602 & 0.616408 \\
\hline $\mathrm{H}$ & -6.749274 & -5.930140 & 0.493921 \\
\hline $\mathrm{H}$ & -6.426258 & -4.406177 & -2.091750 \\
\hline $\mathrm{H}$ & -7.253076 & -5.927547 & -1.826418 \\
\hline $\mathrm{H}$ & -9.203047 & -4.743465 & -0.845963 \\
\hline $\mathrm{H}$ & -8.317898 & -3.227932 & -0.828755 \\
\hline $\mathrm{H}$ & -8.194871 & -3.234471 & -3.315348 \\
\hline $\mathrm{H}$ & -9.127509 & -4.726112 & -3.370333 \\
\hline $\mathrm{H}$ & -11.052024 & -3.584743 & -2.268179 \\
\hline $\mathrm{H}$ & -10.110638 & -2.105815 & -2.110233 \\
\hline $\mathrm{H}$ & -10.832724 & -3.399571 & -4.788766 \\
\hline $\mathrm{H}$ & -9.944542 & -1.894856 & -4.569155 \\
\hline $\mathrm{H}$ & -12.827081 & -2.389377 & -3.659394 \\
\hline $\mathrm{H}$ & -11.922166 & -0.898860 & -3.395914 \\
\hline $\mathrm{H}$ & -12.585204 & -2.083793 & -6.142769 \\
\hline $\mathrm{H}$ & -11.690314 & -0.595975 & -5.864629 \\
\hline $\mathrm{C}$ & 11.961201 & 2.920606 & -1.671303 \\
\hline $\mathrm{C}$ & 10.997713 & 2.710844 & -0.525266 \\
\hline $\mathrm{C}$ & 10.372410 & 1.321521 & -0.538419 \\
\hline $\mathrm{C}$ & 9.390998 & 1.132518 & 0.616776 \\
\hline $\mathrm{C}$ & 8.693842 & -0.223734 & 0.576181 \\
\hline $\mathrm{C}$ & 7.277183 & -0.306707 & 1.088885 \\
\hline $\mathrm{C}$ & 7.626437 & -0.422838 & -0.467936 \\
\hline C & 7.251165 & -1.709489 & -1.115514 \\
\hline $\mathrm{C}$ & 6.788033 & -2.801164 & -0.417027 \\
\hline C & 6.467198 & -2.696583 & 1.018024 \\
\hline $\mathrm{C}$ & 6.623488 & -1.504097 & 1.687838 \\
\hline $\mathrm{C}$ & 5.596742 & -1.060036 & 2.591315 \\
\hline $\mathrm{C}$ & 4.481649 & -1.844143 & 2.851732 \\
\hline $\mathrm{C}$ & 3.175454 & -1.229356 & 2.977541 \\
\hline $\mathrm{C}$ & 2.209095 & -2.116325 & 2.365814 \\
\hline $\mathrm{C}$ & 1.145521 & -1.590056 & 1.648270 \\
\hline $\mathrm{C}$ & 0.757879 & -2.197448 & 0.393471 \\
\hline $\mathrm{C}$ & 0.360393 & -1.143010 & -0.516775 \\
\hline C & 0.663842 & -1.243234 & -1.866410 \\
\hline $\mathrm{C}$ & 1.369690 & -2.405587 & -2.368737 \\
\hline $\mathrm{C}$ & 1.751339 & -3.413273 & -1.49498 \\
\hline
\end{tabular}




\begin{tabular}{|c|c|c|c|}
\hline $\mathrm{C}$ & 1.434639 & -3.309988 & -0.083029 \\
\hline $\mathrm{C}$ & 2.542551 & -3.859350 & 0.668113 \\
\hline $\mathrm{C}$ & 2.922697 & -3.275141 & 1.865413 \\
\hline C & 4.328715 & -3.102906 & 2.171817 \\
\hline C & 5.297467 & -3.507816 & 1.261372 \\
\hline C & 4.894598 & -4.117933 & 0.010408 \\
\hline C & 3.551159 & -4.294231 & -0.279236 \\
\hline C & 3.056915 & -4.026382 & -1.616456 \\
\hline $\mathrm{C}$ & 3.929980 & -3.604277 & -2.606244 \\
\hline $\mathrm{C}$ & 5.337031 & -3.431966 & -2.305177 \\
\hline $\mathrm{C}$ & 5.810294 & -3.674883 & -1.020797 \\
\hline $\mathrm{C}$ & 5.805465 & -2.277079 & -3.023549 \\
\hline C & 6.724222 & -1.427776 & -2.422692 \\
\hline $\mathrm{C}$ & 6.584702 & 0.006393 & -2.561021 \\
\hline $\mathrm{C}$ & 7.027345 & 0.626113 & -1.339734 \\
\hline $\mathrm{C}$ & 6.343421 & 1.717138 & -0.850003 \\
\hline $\mathrm{C}$ & 6.019115 & 1.822582 & 0.585848 \\
\hline $\mathrm{C}$ & 6.393522 & 0.831172 & 1.465647 \\
\hline $\mathrm{C}$ & 5.454235 & 0.373643 & 2.454634 \\
\hline $\mathrm{C}$ & 4.203500 & 0.961496 & 2.583832 \\
\hline $\mathrm{C}$ & 3.038813 & 0.140704 & 2.847111 \\
\hline $\mathrm{C}$ & 1.929090 & 0.693157 & 2.099567 \\
\hline $\mathrm{C}$ & 1.000340 & -0.153072 & 1.512320 \\
\hline $\mathrm{C}$ & 0.519118 & 0.121548 & 0.173899 \\
\hline $\mathrm{C}$ & 0.980459 & 1.232582 & -0.517675 \\
\hline $\mathrm{C}$ & 1.949816 & 2.113934 & 0.096652 \\
\hline $\mathrm{C}$ & 2.413964 & 1.852520 & 1.376460 \\
\hline $\mathrm{C}$ & 3.821552 & 2.016734 & 1.682859 \\
\hline $\mathrm{C}$ & 4.709919 & 2.421913 & 0.694211 \\
\hline $\mathrm{C}$ & 4.220874 & 2.689556 & -0.641331 \\
\hline $\mathrm{C}$ & 2.876090 & 2.540755 & -0.932711 \\
\hline $\mathrm{C}$ & 2.467484 & 1.942500 & -2.186703 \\
\hline $\mathrm{C}$ & 1.302211 & 1.125823 & -1.928728 \\
\hline C & 1.142040 & -0.082505 & -2.590465 \\
\hline $\mathrm{C}$ & 2.141364 & -0.525874 & -3.540100 \\
\hline $\mathrm{C}$ & 2.282431 & -1.962497 & -3.402828 \\
\hline C & 3.533464 & -2.549763 & -3.519843 \\
\hline $\mathrm{C}$ & 4.696509 & -1.726551 & -3.779263 \\
\hline $\mathrm{C}$ & 4.561965 & -0.354860 & -3.911838 \\
\hline $\mathrm{C}$ & 3.256795 & 0.260360 & -3.789751 \\
\hline $\mathrm{C}$ & 3.423959 & 1.523552 & -3.097651 \\
\hline $\mathrm{C}$ & 4.831859 & 1.688459 & -2.796240 \\
\hline $\mathrm{C}$ & 5.529572 & 0.532228 & -3.293403 \\
\hline $\mathrm{C}$ & 5.224578 & 2.253637 & -1.588670 \\
\hline $\mathrm{C}$ & 9.600062 & -1.386081 & 0.879957 \\
\hline $\mathrm{C}$ & 10.274868 & -2.057081 & -0.139741 \\
\hline $\mathrm{C}$ & 11.158315 & -3.089227 & 0.160930 \\
\hline $\mathrm{C}$ & 11.372672 & -3.460583 & 1.484847 \\
\hline
\end{tabular}

\begin{tabular}{|c|c|c|c|}
\hline $\mathrm{C}$ & 10.703070 & -2.794055 & 2.507855 \\
\hline C & 9.822120 & -1.761808 & 2.205335 \\
\hline $\mathrm{C}$ & 13.431589 & 4.445423 & -2.667797 \\
\hline $\mathrm{H}$ & 10.228747 & 3.491438 & -0.585737 \\
\hline $\mathrm{H}$ & 11.538373 & 2.896252 & 0.411133 \\
\hline $\mathrm{H}$ & 9.852408 & 1.170658 & -1.491686 \\
\hline $\mathrm{H}$ & 11.162840 & 0.565555 & -0.495018 \\
\hline $\mathrm{H}$ & 8.649577 & 1.937854 & 0.598512 \\
\hline $\mathrm{H}$ & 9.913155 & 1.212394 & 1.578453 \\
\hline $\mathrm{H}$ & 10.104487 & -1.772195 & -1.174255 \\
\hline $\mathrm{H}$ & 11.676838 & -3.604568 & -0.641721 \\
\hline $\mathrm{H}$ & 12.057966 & -4.269471 & 1.719421 \\
\hline $\mathrm{H}$ & 10.863047 & -3.081221 & 3.542672 \\
\hline $\mathrm{H}$ & 9.292552 & -1.246653 & 3.002817 \\
\hline $\mathrm{H}$ & 13.771488 & 5.463197 & -2.478242 \\
\hline $\mathrm{H}$ & 12.941831 & 4.380542 & -3.642614 \\
\hline $\mathrm{H}$ & 14.274724 & 3.750355 & -2.648364 \\
\hline $\mathrm{O}$ & 12.509054 & 4.145925 & -1.621024 \\
\hline $\mathrm{O}$ & 12.217337 & 2.110616 & -2.530144 \\
\hline $\mathrm{C}$ & -2.388832 & -1.088612 & 4.030778 \\
\hline$S$ & -2.466674 & 0.655695 & 3.882828 \\
\hline $\mathrm{C}$ & -4.190098 & 0.515307 & 3.509114 \\
\hline $\mathrm{C}$ & -4.550429 & -0.877749 & 3.529429 \\
\hline $\mathrm{N}$ & -3.571379 & -1.715170 & 3.789308 \\
\hline $\mathrm{C}$ & -4.961645 & 1.606876 & 3.198644 \\
\hline $\mathrm{C}$ & -4.486544 & 2.926344 & 3.081309 \\
\hline $\mathrm{C}$ & -5.955569 & -1.444745 & 3.337097 \\
\hline $\mathrm{C}$ & -6.653270 & -0.876590 & 2.080771 \\
\hline $\mathrm{C}$ & -1.227146 & -1.757441 & 4.365016 \\
\hline $\mathrm{C}$ & -0.078999 & -1.022010 & 4.763996 \\
\hline $\mathrm{N}$ & 0.822095 & -0.375215 & 5.111871 \\
\hline $\mathrm{C}$ & -5.871196 & -2.972732 & 3.194903 \\
\hline $\mathrm{C}$ & -6.779439 & -1.106004 & 4.596663 \\
\hline $\mathrm{C}$ & -1.162905 & -3.178715 & 4.338453 \\
\hline $\mathrm{N}$ & -1.080487 & -4.335931 & 4.281266 \\
\hline $\mathrm{H}$ & -6.004713 & 1.413527 & 2.994835 \\
\hline $\mathrm{H}$ & -7.393702 & -1.594612 & 1.713267 \\
\hline $\mathrm{H}$ & -7.201215 & 0.048759 & 2.281980 \\
\hline $\mathrm{H}$ & -5.943560 & -0.686207 & 1.272186 \\
\hline $\mathrm{H}$ & -6.885938 & -3.381454 & 3.127763 \\
\hline $\mathrm{H}$ & -5.325264 & -3.247849 & 2.287206 \\
\hline $\mathrm{H}$ & -5.359417 & -3.432978 & 4.041931 \\
\hline $\mathrm{H}$ & -7.794713 & -1.503287 & 4.488735 \\
\hline $\mathrm{H}$ & -6.328941 & -1.551655 & 5.489320 \\
\hline $\mathrm{H}$ & -6.851207 & -0.024868 & 4.755405 \\
\hline $\mathrm{C}$ & -5.223284 & 4.005040 & 2.635708 \\
\hline $\mathrm{C}$ & -6.694345 & 4.044599 & 2.205680 \\
\hline $\mathrm{N}$ & -4.687422 & 5.242094 & 2.483599 \\
\hline
\end{tabular}




\begin{tabular}{|c|c|c|c|}
\hline C & -5.623790 & 6.142948 & 1.935093 \\
\hline C & -6.839043 & 5.488544 & 1.772246 \\
\hline $\mathrm{H}$ & -3.434684 & 3.089704 & 3.293000 \\
\hline & -7.640081 & 3.722001 & 3.376073 \\
\hline C & -6.953128 & 3.133455 & 0.992748 \\
\hline C & -7.919666 & 6.157253 & 1.228055 \\
\hline C & -7.764576 & 7.495358 & 0.859321 \\
\hline C & -6.541355 & 8.137587 & 1.031205 \\
\hline C & -5.441793 & 7.468091 & 1.571381 \\
\hline H & -4.487870 & 7.972900 & 1.681393 \\
\hline $\mathrm{H}$ & -6.431718 & 9.174366 & 0.729387 \\
\hline & -8.598388 & 8.031203 & 0.418798 \\
\hline & -8.866242 & 5.649972 & 1.067031 \\
\hline $\mathrm{H}$ & -7.986874 & 3.262773 & 0.658734 \\
\hline $\mathrm{H}$ & -6.299548 & 3.397790 & 0.157720 \\
\hline $\mathrm{H}$ & -6.801880 & 2.074919 & 1.220941 \\
\hline & -8.675568 & 3.880921 & 3.059559 \\
\hline $\mathrm{H}$ & -7.54 & 2.68 & 193 \\
\hline & -7.440074 & 4.371475 & 4.233273 \\
\hline & -3.277584 & 5.568367 & 2.631222 \\
\hline $\mathrm{C}$ & -2.487271 & 5.227588 & 1.367317 \\
\hline c & -1.007218 & 5.589989 & 1.466865 \\
\hline $\mathrm{C}$ & -0.268649 & 5.366395 & 0.146888 \\
\hline $\mathrm{C}$ & 1.206394 & 5.762648 & 0.195465 \\
\hline $\mathrm{C}$ & 1.873413 & 5.720278 & -1.178655 \\
\hline $\mathrm{C}$ & 3.378340 & 5.979495 & -1.132352 \\
\hline $\mathrm{C}$ & 4.076754 & 5.710663 & -2.465436 \\
\hline $\mathrm{C}$ & 5.594176 & 5.856528 & -2.379444 \\
\hline $\mathrm{H}$ & 6.071167 & 5.656351 & -3.344391 \\
\hline $\mathrm{H}$ & 5.877429 & 6.868032 & -2.065905 \\
\hline & 6.010323 & 5.152514 & -1.648835 \\
\hline $\mathrm{H}$ & -2.882126 & 5.044431 & 3.506224 \\
\hline $\mathrm{H}$ & -3.206882 & 6.635847 & 2.858081 \\
\hline $\mathrm{H}$ & -2.595007 & 4.157080 & 1.157260 \\
\hline $\mathrm{H}$ & -2.943799 & 5.757011 & 0.520944 \\
\hline $\mathrm{H}$ & -0.905922 & 6.644834 & 1.759730 \\
\hline $\mathrm{H}$ & -0.531035 & 4.999980 & 2.262024 \\
\hline & -0.345378 & 4.307411 & -0.140773 \\
\hline $\mathrm{H}$ & -0.773939 & 5.937162 & -0.645158 \\
\hline $\mathrm{H}$ & 1.304668 & 6.772252 & 0.618975 \\
\hline $\mathrm{H}$ & 1.738965 & 5.087021 & 0.879848 \\
\hline $\mathrm{H}$ & 1.392418 & 6.451112 & -1.843738 \\
\hline $\mathrm{H}$ & 1.692213 & 4.736528 & -1.632424 \\
\hline & 3.567181 & 7.015589 & -0.817738 \\
\hline H & 3.829352 & 5.341272 & -0.360116 \\
\hline & 3.679053 & 6.389648 & -3.231317 \\
\hline & 3.829027 & 4.694335 & -2.801122 \\
\hline
\end{tabular}

